\title{
Detecting impending symptom transitions using early warning signals in individuals receiving treatment for depression
}

Marieke A. Helmich ${ }^{1 *}$, Arnout C. Smit ${ }^{1}$, Laura F Bringmann ${ }^{1,2}$, Marieke J. Schreuder ${ }^{1}$, Albertine J. Oldehinkel $^{1}$, Marieke Wichers ${ }^{1}$, Evelien Snippe ${ }^{1}$

${ }^{1}$ University of Groningen, University Medical Center Groningen, Department of Psychiatry, Interdisciplinary Center Psychopathology and Emotion Regulation, P.O. Box 30.001 (CC72), 9700 RB Groningen, the Netherlands

${ }^{2}$ University of Groningen, Faculty of Behavioural and Social Sciences, Department of Psychometrics and Statistics, Grote Kruisstraat 2/1, 9712 TS, Groningen, The Netherlands

*Corresponding author. Email address: marieke.a.helmich@gmail.com

This manuscript was accepted for publication in Clinical Psychological Science on June 14, 2022 and is currently in press. 


\begin{abstract}
Drawing on dynamical systems theory, this study investigated whether within-person detected early warning signals (EWS) in momentary affect preceded critical transitions towards lower levels of depressive symptoms during therapy. Participants were 41 depressed individuals who were starting psychological treatment. Positive and negative affect (high and low arousal) were measured five times a day using ecological momentary assessments over four months ( $\mathrm{M}=521$ observations per individual). Depressive symptoms were assessed weekly over six months. Within-person rising autocorrelation was found for $89 \%$ of individuals with transitions in at least one variable (62.5\% in the no-transition group), and in a consistently higher proportion of the separate variables ( $44 \%$ across affect measures) than for individuals without transitions $(\sim 27 \%)$. Rising variance was found for few individuals, both preceding transitions $(\sim 11 \%)$ and for individuals without a transition $(\sim 12 \%)$. Part of our sample showed critical slowing down, but EWS may have limited value as a personalized prediction method.
\end{abstract}

Keywords: complexity; destabilization; symptom remission; sudden improvement; personalized models; idiographic change; replicated single-subject design; experience sampling method (ESM) 


\section{Background}

Researchers have started drawing on dynamical systems theory for methods to improve their understanding of the processes of change in psychopathology (Cramer et al., 2016; Gelo \& Salvatore, 2016; Hayes \& Andrews, 2020). In part, this renewed interest in dynamical systems is due the rise in studies revealing that changes in psychopathology are often nonlinear (Delignières et al., 2004; Fisher et al., 2011; Hamaker \& Wichers, 2017; Hayes et al., 2007; Helmich et al., 2020b; Hosenfeld et al., 2015; Molenaar \& Campbell, 2009). In the dynamical systems framework, people are viewed as complex systems of interacting components (e.g., behaviors, cognitions and emotions) in which the dynamics of those components can be studied to anticipate impending changes (Boker et al., 2016; Fried \& Robinaugh, 2020; Jeronimus, 2019). Particularly, the phenomenon of 'critical slowing down' may lead to early warning signals (EWS) in the temporal dynamics of the system variables before a critical transition from one dynamically stable state to another (e.g., from a depressed to non-depressed state). When a system critically slows, it destabilizes and gradually becomes less resilient to external shocks (e.g., daily events), which results in more extreme fluctuations (rising variance) and a longer time to return to the equilibrium position (rising autocorrelation). EWS have been described as generic indicators that a system may be losing resilience and nearing a tipping point where the likelihood of a critical transition is higher (Dakos et al., 2014; Scheffer, 2009; Scheffer et al., 2012; Zeeman, 1976). EWS are generic in the sense that they are expected to occur in a wide range of complex dynamical systems (Drake \& Griffen, 2010; Scheffer et al., 2012), and are beginning to be explored in the field of psychiatry (Bos et al., 2022; Dablander et al., 2022; Gijzel et al., 2020; Helmich et al., 2021; Kunkels et al., 2021; Olthof et al., 2020b; Schreuder et al., 2020, 2022, in press; van de Leemput et al., 2014; Wichers et al., 2016, 2020).

An important reason that EWS have attracted such attention from psychopathology researchers is the promise that they may serve as a clinical tool to detect symptom shifts ahead 
of time. Descriptively, they fit with clinical observations: the process of change during psychotherapy is frequently characterized by destabilization and nonlinear patterns (Hayes et al., 2015; Hayes \& Strauss, 1998; Heinzel et al., 2014; Olthof et al., 2020a; Schiepek et al., 2016; Strunk \& Lichtwarck-Aschoff, 2019), such as symptom shifts between therapy sessions known as sudden gains and sudden losses (Aderka et al., 2012; Helmich et al., 2020b; Lutz et al., 2013; Olthof et al., 2020b; Shalom \& Aderka, 2020; Tang \& DeRubeis, 1999), or rapid early response curves over the first few weeks of treatment (Husen et al., 2016; Kelley et al., 2018; Nordberg et al., 2014; Stulz et al., 2007; Vittengl et al., 2016). These nonlinear patterns are often linked to better outcomes (e.g., Haas et al., 2002; Heinzel et al., 2014; Helmich et al., 2020b; Olthof et al., 2020a; Shalom \& Aderka, 2020; Stulz et al., 2007; Vittengl et al., 2016), so using EWS to improve early detection of destabilization and potential shifts could provide therapists clues about their patients' response potential, and allow them to personalize the treatment and timing of interventions accordingly. In fact, based on the synergetics view on psychotherapy, the therapeutic alliance and the safe environment created in the context of therapy may serve as 'boundary conditions' for instability to occur that is more likely to result in positive changes (Schiepek, 2009; Schiepek et al., 2014). Thus, examining the promise of EWS is clinically important, as it may give patient and therapist valuable insight into whether the system is destabilizing and therefore more sensitive to therapeutic input, and significant improvement could be imminent (Hayes \& Andrews, 2020; Schiepek et al., 2017; Strunk \& Lichtwarck-Aschoff, 2019; Wright \& Woods, 2020).

Moreover, studying the occurrence of EWS during therapy is theoretically relevant, as it takes the next step from describing the process of symptom remission from a complex systems angle, to actually testing whether dynamical systems phenomena such as critical slowing down apply to transitions in psychological systems (Bos \& De Jonge, 2014). EWSlike dynamics have shown promise in anticipating shifts in depressive symptoms in a few 
(group-level) studies (Curtiss et al., 2021; Gijzel et al., 2017, 2020; Kuranova et al., 2020; Olthof et al., 2020a; Schiepek, 2009; Schreuder et al., 2020; van de Leemput et al., 2014), which correlated individuals' average autocorrelation or variance in mental states to their average symptom severity. However, to truly test the idea that EWS precede symptom transitions in psychopathology, it is imperative to investigate within-person rises in autocorrelation or variance prior to transitions (Bos \& De Jonge, 2014). To that end, Wichers et al. (2016) first showed that EWS could be used as a personalized predictor of depressive relapse in a single patient, a result they replicated in another patient recently (Wichers et al., 2020). In the context of therapy, Olthof et al. (2020b) identified transitions in time series of daily self-ratings of problem intensity for a large sample of mood disorder patients during treatment. They found that EWS in the form of increased dynamic complexity predicted an increased probability of having a transition in symptom severity in the next four days. These results are very promising, as they show that individually calculated destabilization and withinperson defined transitions were positively associated. However, the study by Olthof et al. (2020b) used a multilevel model, which means this association holds for the average estimated effect of destabilization on the likelihood of having a transition, but their findings do not translate to individualized predictions of imminent change. For that, one needs to test whether a significant within-person rise precedes a transition for each individual separately. Therefore, this study aims to take this next step forward, and test whether transitions toward lower levels of depressive symptoms are consistently preceded by within-person rises in EWS.

Theoretically, we would expect EWS to occur in variables that are central to the system under consideration. A state of depression can be said to be composed of a persistent collection of individual depressive symptoms (e.g., anhedonia, sad mood, lack of energy), which in turn are expressed in shorter-lived feelings (Wichers, 2014; Wichers et al., 2015). In the current study, we therefore look for EWS in the smallest building blocks that contribute to the overall 
course of a depressive disorder (Eronen, 2019; Riese \& Wichers, 2019; Wichers, 2014; Wichers et al., 2015): the moment-to-moment fluctuations in affect measured by ecological momentary assessments (EMA). Notably, the presence and strength of EWS may differ between variables (Boerlijst et al., 2013; Dakos, 2018; Lever et al., 2020; Patterson et al., 2021). Therefore, an important secondary goal in the investigation of EWS in the context of psychiatry is to examine which affective states most reliably anticipate transitions towards reduced symptoms of depression.

Gathering appropriate data is challenging, and only two single-subject studies of EWS prior to depressive relapse have been published (Wichers et al., 2016, 2020). To be able to capture and calculate EWS in affect observations before shifts in depressive symptoms, we need frequent momentary assessments over a period in which clinical change is likely. High resolution time series data (Cabrieto et al., 2018; Dablander et al., 2022; Dakos et al., 2012a; Liu et al., 2015) that captures moment-to-moment variations at a time scale that is short enough to cover the full range of fluctuations in the state of the system (Hamaker et al., 2015; Haslbeck \& Ryan, 2021; Jeronimus, 2019; Kuppens et al., 2010; Van Der Bolt et al., 2021), and over a long enough period to capture the entire state change, is required to detect change in the system dynamics. Therefore, we collected intensive longitudinal data from 41 individuals who were starting treatment for depressive complaints as they entered the study, and were thus likely to show symptom improvement. We gathered four months of 5-times daily EMA affect observations to be able to detect EWS, and six months of weekly depressive symptom assessments for the identification of symptom shifts. As EWS are studied within-system (i.e., within each individual), these 41 individuals each represent a single-subject study and replicated test of our hypothesis.

The current study was uniquely designed to test whether critical slowing down-based EWS consistently occur prior to transitions toward depressive symptom improvement, and is 
the first to test this hypothesis empirically, at the within-person level, for multiple individuals. We aimed to establish whether destabilization in the form of EWS such as increasing trends in lag-1 autocorrelation and variance can be detected in momentary-measured affect before transitions towards improvement in depressive symptoms. We hypothesized that EWS would be most prevalent in variables congruent with the direction of the transition, that is, positive affect variables would be most likely to show changing dynamics leading up to a clinical improvement (van de Leemput et al., 2014; Wichers et al., 2016, 2019, 2020). To provide the clearest picture of how well EWS work as personalized indicators of imminent change, we will also examine the rates of EWS in individuals without transitions. We hypothesized that individuals with clear symptom shifts would have relatively more and stronger EWS than individuals without transitions.

\section{Method}

\section{Sample description}

Individuals with current depressive symptoms who were about to start psychological treatment were recruited for this study. This population was chosen with the expectation that these patients would be likely to show symptom improvements during the study period. To be eligible for participation, individuals needed to be 18 years or older, have current depressive symptoms (a score $\geq 14$ on the Inventory of Depressive Symptomatology Self Report (IDSSR; Rush et al., 2000), and be bound for psychological treatment for depression within one month of the (intended) participation start date. Participants also needed to be capable of following the study procedures and operating a smartphone, have sufficient command of the Dutch language, and be willing and able to give informed consent. Exclusion criteria were chronicity of depressive complaints (persistence $\geq 2$ years), current manic or psychotic symptoms, and a primary diagnosis for personality disorder. Written informed consent was 
obtained from all subjects. All procedures were approved on December 12, 2016 by the Medical Ethical Committee of the University Medical Center Groningen (reg. number: NL58848.04.16). Detailed procedures for this research project are described in Helmich et al. (2020a). We report how we determined our sample size, all data exclusions, all manipulations, and all measures in the study.

The data collection took place between June 2017 and May 2020. The intended sample size was 45-50 participants, to arrive at a sufficient number of patients with a depressive symptom transition who could serve as replications of one another. The final sample consisted

Table 1

Sample characteristics: highest level of education and type of treatment received

\begin{tabular}{|c|c|c|c|c|}
\hline Education & $N$ & $\%$ & & \\
\hline Primary & 2 & 4.9 & & \\
\hline Preparatory vocational & 5 & 12.2 & & \\
\hline General secondary & 6 & 14.6 & & \\
\hline Secondary vocational & 13 & 31.7 & & \\
\hline Higher secondary & 10 & 24.4 & & \\
\hline Higher professional & 17 & 41.5 & & \\
\hline University & 12 & 29.3 & & \\
\hline Treatment & Median & Mean & SD & Range \\
\hline Hours per week & 1.0 & 3.1 & 6.0 & $0.0-19.0$ \\
\hline Duration in weeks & 20 & 25.8 & 16.5 & $0.0-54.0$ \\
\hline \multirow[t]{2}{*}{ Type of treatment received } & \multicolumn{2}{|c|}{ Primary } & \multicolumn{2}{|c|}{ Secondary } \\
\hline & $N$ & $\%$ & $N$ & $\%$ \\
\hline CBT & 15 & 36.6 & 1 & 2.4 \\
\hline Intensive combination & 6 & 14.6 & 0 & 0.0 \\
\hline Combination/eclectic & 4 & 9.8 & 0 & 0.0 \\
\hline Group therapy & 3 & 7.3 & 0 & 0.0 \\
\hline Supportive conversations & 2 & 4.9 & 2 & 4.9 \\
\hline Schema therapy & 2 & 4.9 & 2 & 4.9 \\
\hline Mindfulness & 2 & 4.9 & 0 & 0.0 \\
\hline Psychodynamic therapy & 2 & 4.9 & 0 & 0.0 \\
\hline Systems therapy & 1 & 2.4 & 1 & 2.4 \\
\hline Psychoeducation & 1 & 2.4 & 0 & 0.0 \\
\hline Interpersonal therapy & 1 & 2.4 & 0 & 0.0 \\
\hline EMDR & 1 & 2.4 & 3 & 7.3 \\
\hline Person-centered & 0 & 0.0 & 2 & 4.9 \\
\hline Psychomotor therapy & 0 & 0.0 & 1 & 2.4 \\
\hline $\mathrm{ACT}$ & 0 & 0.0 & 1 & 2.4 \\
\hline
\end{tabular}

Note. Total sample $N=41 . \mathrm{CBT}=$ Cognitive Behavioral Therapy; EMDR $=$ Eye Movement Desensitization and Reprocessing; $\mathrm{ACT}=$ Acceptance and Commitment Therapy 
of 41 participants who completed the six-month study period (for an overview, see Figure 1).

The participants were mostly female $(n=35,85 \%)$, with a mean age of 40.1 years $(S D=14.4$, $\min =19, \max =70$ ), at the time of starting the study. Participants' highest level of completed education is reported in Table 1. We did not collect data on the ethnic and cultural background of our participants, and thus cannot be sure that our sample is representative of demographic groups in the Netherlands.

\section{Figure 1}

Flowchart of the Transitions in Depression (TRANS-ID) Recovery participant inclusion steps.

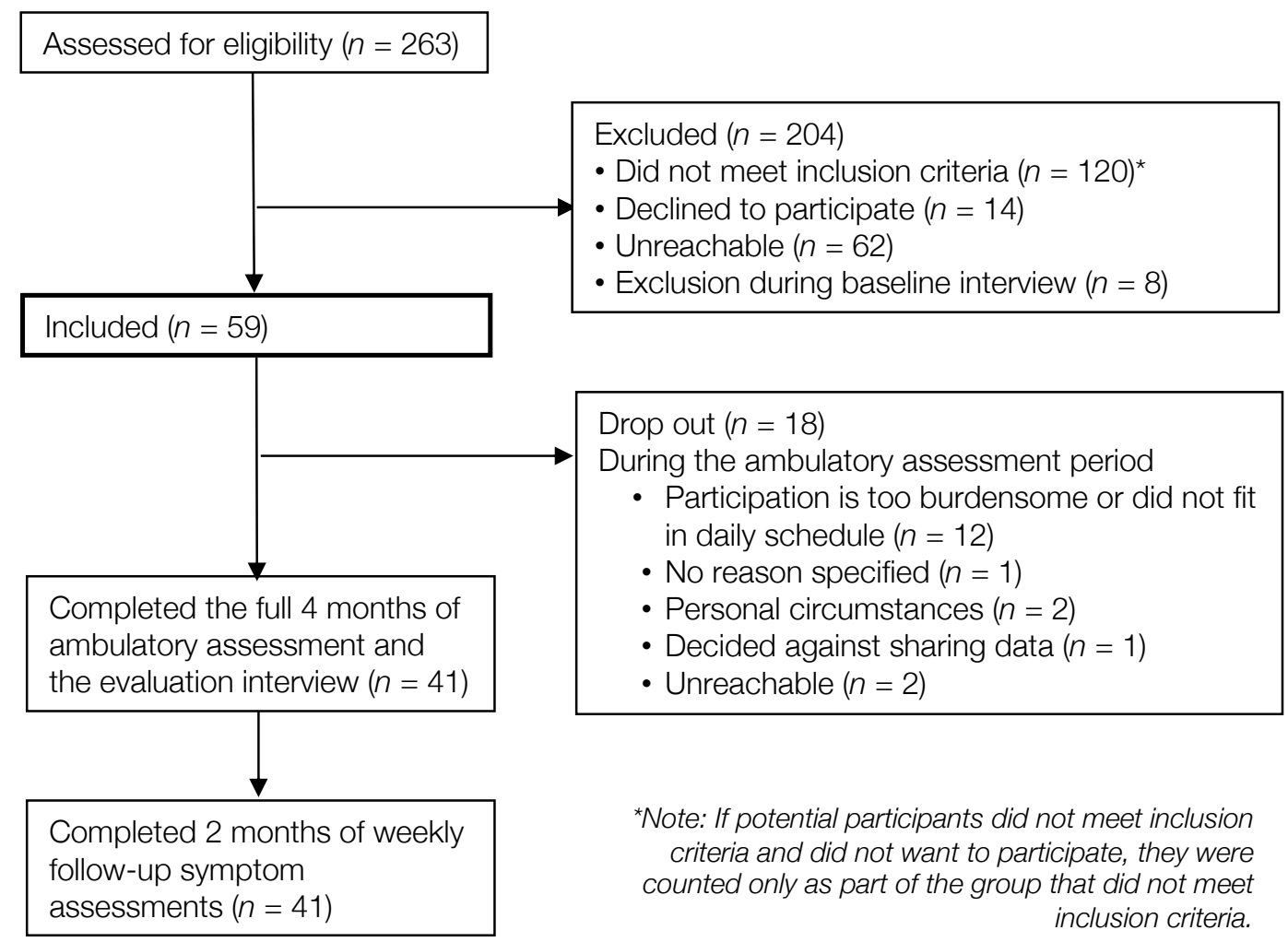




\section{Materials}

\section{Ecological momentary assessment (EMA): momentary mood}

Over a period of four months, participants filled in a 27-item EMA questionnaire about their current feelings, activities and surroundings (available online, see https://osf.io/a8572/) five times a day at set times, with fixed intervals of three hours. Affect was measured with items like "I feel cheerful", and "I feel down", which were answered on a visual analogue scale ranging from "Not at all" to "Very much" (underlying scale ranged from 0 to 100).

With a compliance of $85 \%$, this resulted in $M=522(S D=42, \min =423, \max =591)$ momentary mood measurements per individual, on which the EWS could be calculated $(25,197$ EMA observations in total). To that end, individual items were standardized, person-mean centered and averaged to create four variables based on the affect circumplex (Posner et al., 2005; Yik et al., 1999): Positive Affect (PA) high arousal (“cheerful”, “energetic"), PA low arousal ("content", “at ease"), Negative Affect (NA) high arousal ("restless", "stressed", “irritated"), and NA low arousal (“down", "listless", "tired”). A post hoc confirmatory factor analysis showed adequate fit of the chosen factor structure (see Supplementary Materials section 3 and Table S4).

\section{Depressive symptoms}

Depressive symptoms were measured weekly with fourteen items of the Symptom Checklist90 (SCL-90) Depression subscale (Arrindell \& Ettema, 1981, 2003; Derogatis, 1977). Every weekend, over a period of six months, participants were asked to rate how much in the past week they were bothered by depressive symptoms on a five-point scale from "not at all", to "extremely". With a compliance of $98 \%$, this gave $M=29$ symptom observations per person $(\mathrm{SD}=1.4, \min =23, \max =30)$, and 1,211 assessments in total. The full sixteen-item $($ Dutch $)$ subscale has shown high internal consistency $(\alpha=.91)$ and reliability $\left(\omega_{k}=.93\right)$, as well as a 
strong relationship to the general factor psychological distress (Arrindell \& Ettema, 2003; Smits et al., 2015). For this study, two questions on suicidal ideation were removed from the questionnaire to reduce mental burden for participants ${ }^{1}$. The repeated measurements of the SCL-90 depression subscale were used to identify depressive symptom transitions (see below).

\section{Psychological treatment}

To optimize the chance of capturing transitions in symptoms during the study period, participation was started approximately three weeks before the start of a psychological treatment aimed at reducing depressive symptoms. Participants self-enrolled into a psychological treatment as part of care as usual, completely independent of the study. Information on treatment type, duration and intensity is summarized in Table 1.

\section{Analysis}

This analysis has been preregistered (https://osf.io/xftuq/), meaning that choices regarding the analysis steps (not the study design) were made before the author explored the data. Briefly, we first identified transitions in depressive symptoms (based on weekly symptom data) and then tested (i) whether EWS (based on momentary affect assessments) preceded those transitions and (ii) how often EWS occurred in individuals without transitions. EWS were analyzed for each individual and each affective variable (PA high arousal, PA low arousal, NA high arousal, and NA low arousal) separately using two different methods, namely a movingwindow approach (rises in autocorrelation and variance) and Time-Varying Autoregressive Generalized Additive Modeling (rises in autocorrelation). Missing data were left unaltered, and the calculation of overnight lags was handled by not regressing morning scores on the scores obtained the evening before. We elaborate on each analytical step below.

\footnotetext{
${ }^{1}$ Based on recent studies showing that repeatedly measuring suicidal ideation is feasible (Czyz et al., 2018; Sedano-Capdevila et al., 2021), we now believe omitting these items was an unnecessary precaution.
} 


\section{Transition identification}

In line with descriptions of critical transitions, we aimed to identify symptom reductions that appeared relatively abrupt, were reliable, and remained stable for two weeks (Scheffer, 2009; Wichers et al., 2016; Zeeman, 1976). We did not know the time period over which a critical transition toward improvement would take place, but symptom reductions occurring over time periods of a week to a month have been described as 'rapid' in the clinical literature (Ilardi \& Craighead, 1994; Rubel et al., 2015). Therefore, we used an adapted version of the Reliable Change Index (RCI; Jacobson \& Truax, 1991), the Duration-adjusted RCI (DaRCI; Helmich, 2020), to examine whether symptoms improved reliably over one to maximum four weeks.

The RCI determines the threshold at which the difference between two points is larger than can be expected from measurement error (Jacobson \& Truax, 1991), and is defined as: $R C I=S E_{\text {diff }} * Z$, with $S E_{\text {diff }}=\sqrt{2 *\left(S E_{\mathrm{m}}\right)^{2}}$. Drawing on psychiatric outpatient norm group scores for the SCL-90 depression subscale (Arrindell \& Ettema, 2003), we used a standard error of measurement $\left(S E_{\mathrm{m}}\right)$ of 4.37 for this $\mathrm{study}^{2}$. With a Z-score of 1.96 to set a confidence level of $95 \%$, this yielded $R C I_{95}=6.18 * 1.96=12.11 \approx 13$, indicating that a reliable change between two observations needed to be 13 points or larger (rounded up to preserve the confidence level).

Next, to calculate the thresholds for reliable change over two, three and four weeks, we used the DaRCI. Essentially, this method calculates the critical threshold of change from a starting point ( $\left.t_{\text {start }}\right)$ to the last observation in that period $\left(t_{n}\right)$ while accounting for the longer period between observations. This is done by reducing the RCI to a confidence range around one point, which is then proportionally extended over $n$ points: $\operatorname{DaRCI}=\left(\frac{Z * S E_{\mathrm{diff}}}{2}\right) * n$. This

\footnotetext{
${ }^{2}$ Note that this $S E_{\mathrm{m}}$ is based on all sixteen depression subscale items of the SCL-90, while we only used fourteen items in this study. This meant the threshold we calculated is probably more conservative in identifying transitions.
} 
method allowed us to examine change that is reliable (maintains the confidence threshold) over various durations (for details, see Helmich, 2020). Thus, transitions were identified as reductions of 13, 19, 25 or 31 points over one, two, three or four weeks, respectively (Arrindell \& Ettema, 2003). To summarize, if the time between observations is larger, the size of the change must also be larger to be equally reliable.

To ensure that the identified transitions were not just temporary fluctuations in scores, we added a stability criterion based on the $\mathrm{RCI}_{95}$ that ensured that the mean of the two weeks prior to the first point of a transition $\left(t_{\text {start }-1}, t_{\text {start-2 }}\right)$, and the two weeks after a transition $\left(t_{n+1}\right.$, $t_{n+2}$ ) was at least different by 8.6 points. This threshold accounts for the additional power gained from applying it to a change between two means rather than two single assessments by dividing it by the square root of $n$, such that: $\frac{\mathrm{RCI}_{95}}{\sqrt{2}}=8.6$. For each individual, the earliest transition that met the DaRCI threshold and remained stable the two weeks after the transition was identified. This stability criterion was not preregistered, but it was considered prior to the analyses.

Finally, as this method for identifying transitions has not been tested previously, we examined the $(\mathrm{Da}) \mathrm{RCI}$ at different confidence levels. We took $95 \%$ confidence as a default, and additionally explored transitions identified at $90 \%$ and $99 \%$ confidence.

\section{Moving window method}

In line with the two studies that examined critical slowing down-based EWS within-persons prior to recurrence of depressive symptoms (Wichers et al., 2016, 2020), we used a metricbased moving window approach to examine rises in autocorrelation and variance in the momentary affect measures (cf. Dakos et al., 2012a). Inside a linearly detrended window of 70 observations (two weeks), with outliers winsorized (i.e., extreme values were set to the $5^{\text {th }}$ and $95^{\text {th }}$ percentile), we calculated the values of the lag-1 autocorrelation and the standard deviation of the affect variables within that window. The window was then moved forward 
one observation at a time, and the calculation of the autocorrelation and variance repeated, until the end of the time series (no transition group) or until the transition was reached. The Mann-Kendall test (M-K test, coefficient $\tau$ ) was used to test for a monotonic trend in the entire EWS time series of window estimates of autocorrelation and the variance. To improve the specificity of our test, we maintained a minimally relevant effect size of the trend in the EWS time series of $\tau \geq 0.1$. Specifically, we hypothesized that EWS would be stronger for individuals with compared to individuals without transitions, and therefore, that the $\tau$ coefficients for individuals with a transition would be distributed above zero, and more often have a significant positive trend of $\tau \geq 0.1$ in the EWS time series. In participants without a transition, we would expect the $\tau$-coefficient be distributed around 0 , and therefore to show a lower percentage of significant positive trends.

Sensitivity checks. We explored the effect of the chosen model settings on our findings by rerunning the analysis with the following parameters altered: window sizes of 35, 105 and 140 observations (instead of 70), global detrending (instead of within-window), not removing outliers (instead of winsorizing). We also checked the effect of using only the M-K significance test at $\alpha<.05$ to detect positive trends in EWS, and explored the results when the dependency between window estimates (due to overlapping data) was corrected for, with the modified MK test $\alpha<.05$, as proposed by Hamed and Rao (Hamed \& Rao, 1998). In accordance with our preregistration, the single items "I feel cheerful" and "I feel down" were also analyzed. The results for those variables and the sensitivity checks are reported in the detailed Supplementary Materials.

\section{Time-Varying Autoregressive Generalized Additive Modeling (TV-AR GAM)}

To examine whether the autocorrelation-coefficient rises before a transition, we explored a recently developed method: TV-AR GAM (Bringmann et al., 2017). This method accounts for 
non-stationarity in the data by simultaneously modeling the trend (rather than removing it in preprocessing), allowing both the intercept and autoregressive coefficient to vary over time. The TV-AR GAM uses thin-plate splines to fit smooth curves to the time series, and provides $p$-values for the intercept and autocorrelation parameter, and estimated $95 \%$ credible intervals for the fitted curve. A time-varying trend in the autocorrelation was indicated for curves with (a) a significant autocorrelation parameter, and (b) where a horizontal line could not be fitted within the credible intervals (i.e., the maximum value of the lower limit $>$ the minimum value of the upper limit). To determine if there was truly an overall rise in the trend, three raters (authors M.A.H., L.F.B., and E.S) then visually inspected the diagnostic plots and the modeled smooth curves and decided by consensus on the direction of change - an increase, decrease, or a nonlinear trajectory (e.g., quadratic, cubic, or more complex nonlinear trajectories, apart from those that were very convincingly rising or declining).

Sensitivity checks. As the time series for the non-transitioning group had a higher maximum number of observations $(M=616)$ than the transition group, we performed a sensitivity analysis for the non-transition group with the time series shortened to the average length of time series in the transition-group: 334, 441, and 458 observations, for transitions identified at $90 \%, 95 \%$ and $99 \%$ confidence levels, respectively. 


\section{Results}

\section{Identified transitions}

For nine out of 41 participants $(22 \%)$, we identified reliable symptom reductions with the DaRCI at $95 \%$ confidence. The duration of these symptom transitions varied, with five transitions occurring over one-week, three transitions over two weeks, and one transition over three weeks. On average, transitions took place after 82 days $(S D=29$, range $=51-165)$. With the DaRCI set to $90 \%$ confidence, fourteen individuals (34\%) showed transitions, and at $99 \%$ confidence, six cases (15\%) remained (for the analysis results for the transition groups at $90 \%$ and 99\% confidence, see the Supplementary Material, Tables S2 and S3).

\section{EWS with the moving window method}

Autocorrelation. The results of the moving window method indicated that, of the nine individuals with a transition, $8(89 \%)$ showed EWS in the autocorrelation of at least one affective state $(M=1.78$ EWS per individual, $S D=1.2)$. By comparison, of the 32 individuals without transitions, $20(62.5 \%)$ showed at least one EWS in autocorrelation $(M=1.09$ EWS per individual, $S D=1.17)$.

When investigating EWS in the four variables separately (PA high and low arousal, NA high and low arousal), increases in autocorrelation over time occurred in a higher percentage of individuals with a transition than in individuals without a transition (see Table 1). For individuals with a transition, EWS occurred most often in NA high arousal (55.6\%), and least often in PA low arousal (33.3\%), and for the no-transition group EWS were most often indicated in NA high and low arousal (31.2\% in both), and least often in PA high arousal $(18.8 \%)$. Contrary to our expectations, PA high arousal did not show a higher number of true positive EWS than the other variables, instead showing the lowest sensitivity. 
Variance. Of all nine individuals with a transition, 4 (44\%) showed EWS in the variance of at least one affective state $(M=0.44$ EWS per individual, $S D=0.53)$, as did 8 (25\%) of the 32 individuals without transitions $(M=0.47$ EWS per individual, $S D=0.95)$.

The moving window analysis showed lower proportions of EWS in the variance of the affect measures than the autocorrelation (see Table 1). For some variables, the percentage of EWS appeared to be higher in the transition group than in the non-transition groups (e.g., for PA low there were $3(33 \%)$ EWS in the transition group and $2(6.2 \%)$ in the non-transition group). However, averaging across the four variables revealed no discernible difference between the groups, with $11.1 \%$ true positive EWS in the transition group and $11.7 \%$ false positive EWS in the non-transition group. Again, PA high was not the best performing measure, showing $0 \%$ true positive EWS and $15.6 \%$ false positives in the variance.

Distribution of the $\tau$-coefficients. We expected the Mann-Kendall $\tau$-coefficients to be distributed above zero for individuals with transitions, and around zero in the group without transitions. However, the average $\tau$-coefficients were lower than expected. As can be seen in Table 1 , the average $\tau$-coefficients for the autocorrelation of the different affect measures appeared to be distributed only marginally above zero in the transition group, and below zero for the no-transition group. Only the $\tau$-coefficients of the autocorrelations of PA low and NA high had average values above the $\tau \geq 0.1$ threshold, and showed mostly slightly negative values in the group without transitions. For the variance, the $\tau$-coefficients of all affect measures seemed to be more strongly negative on average, with similar values in both groups (for the distributions of the $\tau$-values, see Figures S1a and S1b in the Supplementary Materials). 
Table 2

Results of the moving window and TV-AR GAM analyses: average $\tau$-values and proportion of early warning signals in the autocorrelation and variance for individuals with and without transitions.

Autocorrelation

Variance

\begin{tabular}{ccccc}
\hline TV-AR GAM & \multicolumn{2}{c}{ Moving Window method } & \multicolumn{2}{c}{ Moving Window method } \\
\hline EWS & EWS & $\tau$ & EWS & $\tau$ \\
$\%(\mathrm{~N})$ & $\%(\mathrm{~N})$ & Mean $(\mathrm{SD})$ & $\%(\mathrm{~N})$ & Mean $(\mathrm{SD})$
\end{tabular}

Transition $(\mathrm{N}=9)$

$\begin{array}{llllll}\text { PA high } & 0 \%(0) & 33.3 \%(3) & -0.09(0.37) & 0 \%(0) & -0.35(0.27) \\ \text { PA low } & 11.1 \%(1) & 44.4 \%(4) & 0.13(0.24) & 33.3 \%(3) & -0.24(0.42) \\ \text { NA high } & 11.1 \%(1) & 55.6 \%(5) & 0.21(0.36) & 11.1 \%(1) & -0.35(0.35) \\ \text { NA low } & 0 \%(0) & 44.4 \%(4) & -0.01(0.39) & 0 \%(0) & -0.36(0.24) \\ \text { verall mean } & \mathbf{5 . 6 \%} & \mathbf{4 4 . 4 \%} & \mathbf{0 . 0 6} & \mathbf{1 1 . 1 \%} & -\mathbf{0 . 3 3}\end{array}$

No transition $(\mathbf{N}=32)$

$\begin{array}{clllll}\text { PA high } & 3.1 \%(1) & 18.8 \%(6) & -0.08(0.2) & 15.6 \%(5) & -0.28(0.42) \\ \text { PA low } & 3.1 \%(1) & 28.1 \%(9) & -0.07(0.26) & 6.2 \%(2) & -0.37(0.32) \\ \text { NA high } & 3.1 \%(1) & 31.2 \%(10) & -0.06(0.24) & 9.4 \%(3) & -0.27(0.36) \\ \text { NA low } & 3.1 \%(1) & 31.2 \%(10) & -0.05(0.25) & 15.6 \%(5) & -0.27(0.35) \\ \text { Overall mean } & \mathbf{3 . 1 \%} & \mathbf{2 7 . 3 \%} & \mathbf{- 0 . 0 7} & \mathbf{1 1 . 7 \%} & \mathbf{- 0 . 3 0}\end{array}$

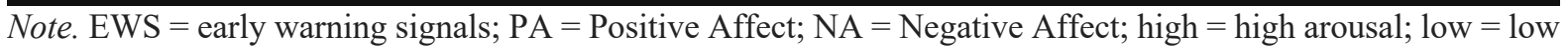
arousal; TV-AR GAM = time-varying autoregressive generalized additive model; $\mathrm{SD}=$ standard deviation; $\tau=$ coefficient of the Mann-Kendall trend test. The results for the TV-AR GAM represent cases where the modeled smooth curve was significantly increasing. The results for the Moving Window method are based on the analysis of transitions at 95\% confidence, where individual time series were winsorized, locally detrended within windows of 70 observations, and the number of early warning signals based on cases with $\tau \geq .1$

Sensitivity checks. For the autocorrelation, the finding that proportionally more EWS were found in the transition group was robust across all the altered model settings for the moving window analysis. The changes in the number of EWS for the autocorrelation found with these altered model settings were similar for people with a transition (difference in number of EWS compared to the main analysis: Median $=0$, first quartile $(\mathrm{Q} 1)=-1$, third quartile $(\mathrm{Q} 3)$ $=0.75)$ and for people without a transition $($ Median $=0, \mathrm{Q} 1=-1, \mathrm{Q} 3=1)$. Increasing the 
window size to 105 or 140 observations yielded an improvement of the accuracy for this sample, at 50\% and 58\% true positives and 33 and $31 \%$ false positives, respectively.

For the variance, the lack of a proportional difference in EWS between the transition and non-transition group was consistent across the sensitivity checks. For the altered analysis settings, similar changes in the number of indicated EWS were found for the transition group (difference in number of EWS compared to the main analysis: Median $=0, \mathrm{Q} 1=-0.75, \mathrm{Q} 3=$ 0), and the people with no transition (Median = $, \mathrm{Q} 1=0, \mathrm{Q} 3=1$; see also Tables S1a and S1b in the Supplementary Materials). None of the altered model settings for the variance yielded notably improved accuracy.

As a post hoc sensitivity check, we inverted our transition criteria to identify participants with transitions towards higher levels of symptoms. The DaRCI thresholds over 1-4 weeks at $95 \%$ confidence were used to identify reliable, sudden symptom increases. The inverted stability criterion tested if the mean of two weeks prior to the shift was at least 8.6 points lower than the mean of the two weeks after the identified transition. This revealed that four cases from the non-transition group experienced a transition to higher symptom levels, and one case with a transition towards lower symptom levels also had a reliable worsening of symptoms that occurred earlier than their reliable improvement. Removing these individuals from the summary findings and recalculating the EWS per affect measure over the remaining individuals did not lead to strongly different results (for more detail see page 8 and Table S1a and S1b in the Supplementary Materials).

\section{EWS with the TV-AR GAM}

The trajectories fitted by the TV-AR GAM indicated that, across the four affect variables, the proportion of significant increases in the autocorrelation was comparable between the transition group $(M=5.6 \%)$ and the non-transition group $(M=3.1 \%$; see Table 1). Significant decreases 
and nonlinear trajectories were indicated more often than increases in both groups, with respective means of $8.3 \%$ and $27.8 \%$ in the transition group, and $M=9.4 \%$ and $25.8 \%$ in the non-transition group.

Sensitivity checks. Reducing the time series length for the non-transitioning individuals resulted in fewer cases with a significantly time-varying smooth curve (approximately $30 \%$ compared to $40 \%$ at full length), although the proportion of significant increases in autocorrelation remained similar (2.3\%, compared to $3.1 \%$ at full length). With transitions identified at $90 \%$ or $99 \%$ confidence, the number of true-positive significant rises in autocorrelation was comparable to the results at 95\% confidence (although at $99 \%$ confidence, no EWS remained in the transition group), as did the number of false positive EWS (see Table S3 in the Supplementary Materials).

\section{Exploratory findings}

In addition to answering the main research question, we also aimed to investigate ways to potentially improve the balance of true and false positives of our results. We explored whether we could improve the sensitivity of the moving window analysis by separating participants according to a minimum cumulative number of EWS. Combining the results of the variance and autocorrelation for PA high, PA low, NA high and NA low showed that selecting individuals with a minimum of two EWS (out of a possible eight) improved the sensitivity, with $77.8 \%$ true positives compared to $44.4 \%$ in the main analysis, but also resulted in an increase in false positives from $27.3 \%$ in the main results, to $40.6 \%$ (for more detail, see Figures S2 and S3 in the Supplementary Materials).

Moreover, we explored different minimally relevant effect sizes by varying the threshold $\tau$-value for the moving window analysis between 0 and 0.5 . Our results did not reveal a clear cut-off value that improved the accuracy for all variables (see Figure S4 in the 
Supplementary Material). Overall, lowering or raising the $\tau$-threshold led to a change the number of true positives that was approximately matched by the change in false positives.

\section{Discussion}

This is the first paper to provide an empirical investigation of whether critical slowing downbased EWS in the form of increasing trends in lag-1 autocorrelation and variance could be detected in individual affect time series in the period before a transition toward depressive symptom improvement. We examined the prevalence of EWS prior to the symptom transitions identified for nine individuals, as well as the rate of false positive EWS in the time series of 32 individuals without transitions. We found that rising autocorrelation was present in at least one of the affect measures for eight out of nine (89\%) individuals with a transition, and for twenty out of the $32(62.5 \%)$ individuals without transitions. When examining the affect measures separately in the moving window analysis, we found that EWS in the form of rising autocorrelation were present in proportionally more individuals with a transition $(\sim 44 \%)$ than without a transition $(\sim 27 \%)$. A rise in the variance was found in at least one variable for four out of nine (44\%) individuals with a transition, and for eight of the $32(25 \%)$ individuals without transitions. The proportion of EWS in the variance for the separate affect measures showed similar rates for individuals with a transition $(\sim 11 \%)$ and without a transition $(\sim 12 \%)$ and thus did not show the same differentiation between the groups. Furthermore, the TV-AR GAM analysis indicated at most one individual with a significant increase in autocorrelation for each of the four affect measures, in both the group with transitions $(\sim 5.6 \%)$ and in the notransition group $(\sim 3.1 \%)$. In our extensive sensitivity checks, the findings for our analyses were robust. This strengthens our conclusion that the moving-window detected autocorrelation results were in the hypothesized direction, and the process of remission from depression showed signs of critical slowing down in part of the individuals in our sample. 
Looking at the broad pattern of our results, our findings correspond reasonably well to earlier studies of EWS in depression (Curtiss et al., 2021; Olthof et al., 2020b; Wichers et al., 2016, 2020). Like the single-subject studies by Wichers et al. (2016, 2020), our results showed that within-person rises in autocorrelation occurred for some of the participants before a transitions in depressive symptoms - in our sample, this also occurred relatively more often for participants with a transition than for participants with no transition. Unlike those studies by Wichers et al. (2016, 2020), however, we did not find rising variance as an EWS. Furthermore, our finding that EWS occurred before symptom transitions during therapy is in line with the findings by Olthof et al. (2020b), who found that critical fluctuations had a higher probability of occurring in the four days before a sudden gain or loss. However, critical fluctuations are a measure of the spread of scores, like the variance, which did not function as an EWS in our study. The difference in findings may be explained by the fact that we looked at a different population than Wichers et al. (2016, 2020; individuals showing depressive symptom remission, rather than recurrence), and a much longer time period than Olthof et al. (2020b) for the rising fluctuations (over weeks or months, as opposed to days). Yet, taking in the broader evidence of all these studies, including the current study: significant destabilization in the form of rising autocorrelation could be identified prior to transitions in symptoms in the affect measures of depressed patients. In our sample, we found signs of critical slowing down, i.e., a rise in autocorrelation in at least one of the affect measures, for eight of the nine individual systems, which was in line with our hypothesis. This suggests that for those individuals the process of depressive remission showed the nonlinearity one would expect from a dynamic system, particularly in the context of therapy (Hayes \& Andrews, 2020; Hayes \& Strauss, 1998; Heinzel et al., 2014; Schiepek, 2009; Shapiro, 2015; Strunk \& Lichtwarck-Aschoff, 2019).

Although almost all individuals with transitions showed EWS in at least one affect measure, looking at the separate affect measures presents a less consistent picture of critical 
slowing down in our study. Each variable showed EWS for only a few people in the transition group, and, contrary to what we hypothesized, PA high arousal did not turn out to be the most sensitive affect measure to predict of symptom improvements. No single affect variable showed EWS for more than five people with transitions. Thus, EWS were not 'generic' indicators of impending symptom change when considering the variables separately. Theoretical works in ecology have found that variables can be 'silent' and lack EWS when they do not change in the same direction as the destabilization of the main system (Boerlijst et al., 2013; Dakos, 2018; Lever et al., 2020; Patterson et al., 2021). That is, perhaps the heterogeneity of which variables showed EWS was due to the fact that the destabilization occurred in different depressive symptoms for different people. By that reasoning, PA high arousal may have shown fewer EWS if destabilization occurred more strongly in depressive symptoms that do not correlate highly with positive experiences (cheerfulness, feeling energetic; cf. Barrett \& Russell, 1998; Schreuder et al., 2020, 2022; Wichers et al., 2019).

False negatives may also have occurred due to the nature of transitions in our study. Based on theory, critical slowing down and its corresponding EWS would be expected to occur primarily before critical transitions or shifts between states in systems that are dynamically stable around a single point and are slowly losing resilience (Scheffer, 2009; Zeeman, 1976). It is possible that the transitions we detected did not follow this pattern, for example, because the states prior to and after the transition were not sufficiently stable or because the transition was not preceded by a gradual accumulation of instability (Boettiger et al., 2013). The latter may happen if a transition was triggered by a large push (e.g., a psychologically impactful event) rather than gradual destabilization of the system (Hastings \& Wysham, 2010; Nazarimehr et al., 2020; Van Der Bolt et al., 2021; van Nes et al., 2016). Thus, it could be that only a subset of individuals in our sample met the theoretical requirements for detecting EWS, 
or that only some of the affect measurements picked up on the changing dynamics, which may have contributed to a lower sensitivity of EWS.

Furthermore, per affect measure, there were six to ten individuals without transitions who showed false positive EWS in our study. A potential explanation is that those individuals without transitions still experienced a destabilizing influence from entering therapy, and thus also showed changes in the temporal dynamics of their affect during the observation period, even if this did not precede a clear discontinuous symptom transition (or end in a change, at all). This interpretation is supported by the fact that, when we lowered our DaRCI confidence level to $90 \%$, the number of true positive EWS increased (from 3-5, to 5-7 EWS per affect measure), while the false positives decreased (from 6-10, to 5-9 EWS per affect measure; see Supplementary Table S2). This suggests that some false positive EWS in the main analysis were likely due to individuals who did experience a relevant symptom improvement, but for whom the transition criteria at the $95 \%$ confidence level were not met, or the shift was not maintained. Our transition criteria were conservative and selected only the most convincing transitions to rule out symptom changes caused by situational factors (which would not be preceded by accumulating instability). Smaller shifts and unmaintained transitions (e.g., flickering) may have been categorized as belonging to the no-transition group, thereby potentially inflating the rate of false positives, and underestimating the number of true positives. Further explanations are derived from other fields, where critical slowing down has also been shown to occur before gradual transitions (Drake \& Griffen, 2010; Kéfi et al., 2013), as well as in periods with no significant system change (Kéfi et al., 2013; Nazarimehr et al., 2020). However, most of these EWS studies have been conducted in an ecological context, with very different data, and it is hard to tell which of the processes that influence the finding of EWS in other fields translate to our ability to detect EWS in psychological processes. 
The accuracy of our moving-window detected autocorrelation analyses was not high enough to warrant any claims about clinical utility at this stage. Our exploratory analyses indicated that there was room to improve the method's performance, as we found improved accuracy for larger window sizes and when looking at the cumulative number of EWS within individuals (a minimum number of EWS threshold also worked well in two studies of EWS before depressive symptom recurrence, see Smit et al., n.d.; Wichers et al., 2020). Moreover, single predictors are rarely $100 \%$ accurate in isolation, and EWS may provide complementary information to early response indicators such as severity of depression (Allen et al., 2019; de Vries et al., 2019; Geschwind et al., 2011). Gaining a better understanding of which participants are likely to show detectable EWS could also help improve the sensitivity of this method, for instance by examining the effect of boundary conditions, such as a good therapeutic alliance, on the absence or presence of transitions and EWS during therapy (Schiepek et al., 2014).

The results for the variance indicated a similar prevalence of EWS in individuals with and without transitions. In fact, most people in our study (with and without transitions) showed a negative trend in the variance over time. A clinical explanation for these downward trends in variance relates to our study population: given that our participants were entering treatment for depressive symptoms, they may have shown higher instability at the start of the observation period due to the destabilizing influence of entering therapy (and because high levels of variability are associated with higher levels of depression; Bos et al., 2018; Peeters et al., 2006; Thompson et al., 2012). As they improved under the influence of therapy, their emotional responses may have become less volatile, and thus an overall downward trend in variance may have emerged in their time series. Another, practical reason for the declines in the variance is that participants may have gotten more habituated to filling out the EMA questionnaires, and thus used less and less of the available scale. Finally, previous EWS studies have also indicated that the variance may show decreases when less data is available and is a less robust as an 
indicator of impending change compared to autocorrelation (Curtiss et al., 2021; Dakos et al., 2012b; van de Leemput et al., 2014; Wichers et al., 2020).

Even though the autocorrelation worked reasonably well as an EWS with the moving window method, the more conservative TV-AR GAM did not show the same results.(cf. Schreuder et al., in press) Possibly, this approach was not optimally suited for the purposes of testing for an overall rise in autocorrelation. Instead, the TV-AR GAM results highlighted the high degree of nonlinearity in the autocorrelation of affect time series in both groups. While the $\mathrm{M}-\mathrm{K}$ test may detect an overall significant trend over time even if the rise in autocorrelation or variance starts later in the time series, the GAM method is aimed at testing the overall shape of the data, and may instead identify similarly late rises as a curving, nonlinear pattern. We do not know over what time period a rise in EWS would be expected in our sample, but a method that allows significant rises to be detected over shorter periods (e.g., a few weeks prior to a transition), may provide an improved rate of EWS detection.

Another reason the TV-AR GAM may have resulted in lower numbers of EWS, is that this method requires a large amount of data to fit credible intervals that were narrow enough to meet our strict change criterion (the maximum value of the lower bound being larger than the lowest value of the upper bound). Furthermore, the TV-AR GAM is generally conservative and biased to detect zero change if little data is available (cf. Bringmann et al., 2017; Haslbeck et al., 2021; Wood, 2017). While determining the direction of change through visual inspection, we noted that many individuals who showed linear change had confidence bounds that were too wide to identify an increase with certainty. The effect of (in)sufficient data on the TV-AR GAM findings was evident from the sensitivity check in the no-transition group: when the time series were shortened (e.g., from, on average, 616 to 411 observations in the main analysis), fewer significant smooth curves were found, and particularly the number of decreases were 
reduced. It thus seems that the available time series length may have played a limiting role in our ability to detect EWS with this method.

It is difficult to determine to what extent our findings generalize beyond our sample. There were too few individuals with a transition to reliably test whether the proportional differences between the group with a transition and the group without a transition were significant. Future studies should aim for larger between-persons sample sizes to allow for statistical comparison of the groups. Additionally, we based our EWS on critical slowing down, but the within-person predictive value of other types of EWS, such as flickering (Dakos et al., 2013), or the synergetics-based dynamic complexity and permutation entropy (Olthof et al., 2020b; Schiepek et al., 2020) would be worth exploring in future research.

This study was foremost a proof of principle to see if critical slowing down-based EWS could be found in individual time series of people receiving treatment for depressive symptoms. Our study was designed with the specific intent to detect rising autocorrelation and variance in individual time series, with several months intensive longitudinal within-person affect and symptom measurements (totaling 26,408 observations) gathered during a period where symptom improvement was likely. The compliance of $85 \%$ was rather good for an EMA study of this length and intensity, though we cannot be sure of if and how missingness affected the results. Given that our population differed from the previous studies of within-person EWS that looked at relapse in depression (Wichers et al., 2016, 2020), and taking into account the new insights from recent studies (Dablander et al., 2022; Helmich et al., 2021; Schreuder et al., 2020), we preregistered and conducted a very thorough analysis with a range of sensitivity checks. Because of this, we were able to discern the broad pattern of how EWS occurred in our dataset and can more confidently state that our findings were robust. 


\section{Conclusions}

EWS in the form of a rise in the autocorrelation in NA high arousal, NA low arousal, PA high arousal, and PA low arousal preceded symptom transitions toward depressive symptom improvement in part of the participants and occurred more often for individuals with transitions than for individuals without transitions. Almost all individuals $(89 \%)$ with a transition had EWS in autocorrelation for at least one the affect measures, compared to about two-thirds of the participants without transitions. EWS based on the variance of affect did not systematically precede symptom transitions. It is too early to claim any clinical utility of EWS in the context of psychotherapy. However, this study provides much-anticipated empirical evidence that the process of symptom remission can follow dynamical systems principles, beyond a post hoc explanation of nonlinear patterns in psychopathology. 


\section{Declarations}

\section{List of abbreviations}

DaRCI: Duration-adjusted Reliable Change Index

EMA: Ecological Momentary Assessment

EWS: Early warning signals

M-K test: Mann-Kendall test

NA: Negative Affect

PA: Positive Affect

SCL-90: Symptom Checklist-90

TV-AR GAM: Time-Varying Autoregressive Generalized Additive Model

\section{Ethics approval and consent to participate}

Written informed consent was obtained from all subjects. All procedures were approved on December 12, 2016 by the Medical Ethical Committee of the University Medical Center Groningen (reg. number: NL58848.04.16).

\section{Availability of data and materials}

The datasets analyzed in the current study are not publicly available due to the sensitive nature of the data (a small sample of patient data with many observations within patients that cannot be fully anonymized), but are available from the corresponding author on reasonable request.

\section{Funding}

This project has received funding from the European Research Council (ERC) under the European Union's Horizon 2020 research and innovative program (ERC-CoG-2015; No 681466 to M. Wichers) and the Netherlands Organisation for Health Research and Development (ZonMw Off Road; project 451001029 to E. Snippe). The funding sources were not involved in the preparation of this manuscript.

\section{Author Contributions}

Conceptualization: M.A.H., M.W. and E.S.; Data curation: M.A.H., L.F.B. and E.S.; Formal analysis: M.A.H.; Funding acquisition: M.W.; Investigation: M.A.H.; Methodology: M.A.H., A.C.S., M.W. and E.S.; Project administration: M.A.H., M.W. and E.S.; Software: M.A.H., A.C.S., M.J.S. and L.F.B.; Supervision: L.F.B., A.J.O., M.W. and E.S.; Visualization: M.A.H. and M.J.S.; Writingoriginal draft: M.A.H.; Writing - review \& editing: A.C.S., L.F.B., M.J.S., A.J.O. and E.S.

\section{Acknowledgements}

We would like to thank Elly Sol, Margriet Brandsma, Abel Busz, René de Vries and Anneke HolSteegstra for their invaluable help with the data collection, and all the mental health professionals and clinics for their assistance with participant recruitment. We also wish to acknowledge the insightful input from members of the larger TRANS-ID team, including Yoram K. Kunkels, Robin N. Groen, Harriette Riese, Sandip V. George and Fionneke M. Bos. Finally, we are especially thankful to all who individuals participated in the study and contributed their data. 


\section{References}

Aderka, I. M., Nickerson, A., Bøe, H. J., \& Hofmann, S. G. (2012). Sudden gains during psychological treatments of anxiety and depression: A meta-analysis. Journal of Consulting and Clinical Psychology, 80(1), 93-101. https://doi.org/10.1037/a0026455

Allen, N. B., Nelson, B. W., Brent, D., \& Auerbach, R. P. (2019). Short-term prediction of suicidal thoughts and behaviors in adolescents: Can recent developments in technology and computational science provide a breakthrough? Journal of Affective Disorders, 250, 163-169. https://doi.org/10.1016/j.jad.2019.03.044

Arrindell, W. A., \& Ettema, J. H. M. (1981). Dimensional structure, reliability and validity of the Dutch version of the Symptom Checklist (SCL-90): Data based on a phobic and a 'normal' population. [Dutch]. Nederlands Tijdschrift Voor de Psychologie En Haar Grensgebieden, 36(2), 108.

Arrindell, W. A., \& Ettema, J. H. M. (2003). SCL-90: Manual to a multidimensional psychopathologyindicator [SCL-90: Handleiding bij een multidimensionele psychopathologie-indicator] (Pearson, Ed.; 2nd ed.). Swets \& Zeitlinger.

Barrett, L. F., \& Russell, J. A. (1998). Independence and bipolarity in the structure of current affect. Journal of Personality and Social Psychology, 74(4), 967-984. https://doi.org/10.1037//0022-3514.74.4.967

Boerlijst, M. C., Oudman, T., \& Roos, A. M. de. (2013). Catastrophic collapse can occur without early warning: Examples of silent catastrophes in structured ecological models. PLOS ONE, 8(4), e62033. https://doi.org/10.1371/journal.pone.0062033

Boettiger, C., Ross, N., \& Hastings, A. (2013). Early warning signals: The charted and uncharted territories. Theoretical Ecology, 6(3), 255-264. https://doi.org/10.1007/s12080-013-0192-6

Boker, S. M., Staples, A. D., \& Hu, Y. (2016). Dynamics of change and change in dynamics. Journal for Person-Oriented Research, 2(1-2), 34-55. https://doi.org/10.17505/jpor.2016.05

Bos, E. H., \& De Jonge, P. (2014). 'Critical slowing down in depression' is a great idea that still needs empirical proof. Proceedings of the National Academy of Sciences, 111(10), E878-E878. https://doi.org/10.1073/pnas.1323672111

Bos, E. H., de Jonge, P., \& Cox, R. F. A. (2018). Affective variability in depression: Revisiting the inertia-instability paradox. British Journal of Psychology. https://doi.org/10.1111/bjop.12372 
Bos, F. M., Schreuder, M. J., George, S. V., Doornbos, B., Bruggeman, R., van der Krieke, L., Haarman, B. C. M., Wichers, M., \& Snippe, E. (2022). Anticipating manic and depressive transitions in patients with bipolar disorder using early warning signals. International Journal of Bipolar Disorders, 10(1), 12. https://doi.org/10.1186/s40345022-00258-4

Bringmann, L. F., Hamaker, E. L., Vigo, D. E., Aubert, A., Borsboom, D., \& Tuerlinckx, F. (2017). Changing dynamics: Time-varying autoregressive models using generalized additive modeling. Psychological Methods, 22(3), 409-425. https://doi.org/10.1037/met0000085

Cabrieto, J., Adolf, J., Tuerlinckx, F., Kuppens, P., \& Ceulemans, E. (2018). Detecting longlived autodependency changes in a multivariate system via change point detection and regime switching models. Scientific Reports, 8(15637), 1-15. https://doi.org/10.1038/s41598-018-33819-8

Cramer, A. O. J., van Borkulo, C. D., Giltay, E. J., van der Maas, H. L. J., Kendler, K. S., Scheffer, M., \& Borsboom, D. (2016). Major depression as a complex dynamic system. PLoS One, 11(12), [e0167490]. https://doi.org/10.1371/journal.pone.0167490

Curtiss, J. E., Mischoulon, D., Fisher, L. B., Cusin, C., Fedor, S., Picard, R. W., \& Pedrelli, P. (2021). Rising early warning signals in affect associated with future changes in depression: A dynamical systems approach. Psychological Medicine, 1-9. https://doi.org/10.1017/S0033291721005183

Czyz, E. K., King, C. A., \& Nahum-Shani, I. (2018). Ecological assessment of daily suicidal thoughts and attempts among suicidal teens after psychiatric hospitalization: Lessons about feasibility and acceptability. Psychiatry Research, 267, 566-574. https://doi.org/10.1016/j.psychres.2018.06.031

Dablander, F., Pichler, A., Cika, A., \& Bacilieri, A. (2022). Anticipating critical transitions in psychological systems using early warning signals: Theoretical and practical considerations. Psychological Methods, 1-30. https://doi.org/10.1037/met0000450

Dakos, V. (2018). Identifying best-indicator species for abrupt transitions in multispecies communities. Ecological Indicators, 94, 494-502. https://doi.org/10.1016/j.ecolind.2017.10.024

Dakos, V., Carpenter, S. R., Brock, W. A., Ellison, A. M., Guttal, V., Ives, A. R., Kéfi, S., Livina, V., Seekell, D. A., van Nes, E. H., \& Scheffer, M. (2012a). Methods for detecting early warnings of critical transitions in time series illustrated using simulated ecological data. PLoS One, 7(7). https://doi.org/10.1371/journal.pone.0041010 
Dakos, V., Carpenter, S. R., van Nes, E. H., \& Scheffer, M. (2014). Resilience indicators: Prospects and limitations for early warnings of regime shifts. Philosophical Transactions of the Royal Society B: Biological Sciences, 370(1659), 2013026320130263. https://doi.org/10.1098/rstb.2013.0263

Dakos, V., Van Nes, E. H., D’Odorico, P., \& Scheffer, M. (2012b). Robustness of variance and autocorrelation as indicators of critical slowing down. Ecology, 93(2), 264-271. https://doi.org/10.1890/11-0889.1

Dakos, V., van Nes, E. H., \& Scheffer, M. (2013). Flickering as an early warning signal. Theoretical Ecology, 6(3), 309-317. https://doi.org/10.1007/s12080-013-0186-4

de Vries, Y. A., Roest, A. M., Bos, E. H., Burgerhof, J. G. M., van Loo, H. M., \& de Jonge, P. (2019). Predicting antidepressant response by monitoring early improvement of individual symptoms of depression: Individual patient data meta-analysis. The British Journal of Psychiatry, 214(1), 4-10. https://doi.org/10.1192/bjp.2018.122

Delignières, D., Fortes, M., \& Ninot, G. (2004). The fractal dynamics of self-esteem and physical self. Nonlinear Dynamics, Psychology, and Life Sciences, 8(4), 479-510.

Derogatis, L. R. (1977). SCL-90-R: administration, scoring and procedures manual-I for the R(evised) version. John Hopkins University School Medicine.

Drake, J. M., \& Griffen, B. D. (2010). Early warning signals of extinction in deteriorating environments. Nature, 467(7314), 456-459. https://doi.org/10.1038/nature09389

Eronen, M. I. (2019). The levels problem in psychopathology. Psychological Medicine, 1-7. https://doi.org/10.1017/S0033291719002514

Fisher, A. J., Newman, M. G., \& Molenaar, P. C. M. (2011). A quantitative method for the analysis of nomothetic relationships between idiographic structures: Dynamic patterns create attractor states for sustained posttreatment change. Journal of Consulting and Clinical Psychology, 79(4), 552-563. https://doi.org/10.1037/a0024069

Fried, E. I., \& Robinaugh, D. J. (2020). Systems all the way down: Embracing complexity in mental health research. BMC Medicine, 18(1), 205. https://doi.org/10.1186/s12916020-01668-w

Gelo, O. C. G., \& Salvatore, S. (2016). A dynamic systems approach to psychotherapy: A metatheoretical framework for explaining psychotherapy change processes. Journal of Counseling Psychology, 63(4), 379-395. https://doi.org/10.1037/cou0000150

Geschwind, N., Nicolson, N. A., Peeters, F., van Os, J., Barge-Schaapveld, D., \& Wichers, M. (2011). Early improvement in positive rather than negative emotion predicts remission 
from depression after pharmacotherapy. European Neuropsychopharmacology, 21(3), 241-247. https://doi.org/10.1016/j.euroneuro.2010.11.004

Gijzel, S. M. W., Rector, J., van Meulen, F. B., van der Loeff, R. S., van de Leemput, I. A., Scheffer, M., Olde Rikkert, M. G. M., \& Melis, R. J. F. (2020). Measurement of dynamical resilience indicators improves the prediction of recovery following hospitalization in older adults. Journal of the American Medical Directors Association, 21(4), 525-530.e4. https://doi.org/10.1016/j.jamda.2019.10.011

Gijzel, S. M. W., van de Leemput, I. A., Scheffer, M., Roppolo, M., Olde Rikkert, M. G. M., \& Melis, R. J. F. (2017). Dynamical resilience indicators in time series of self-rated health correspond to frailty levels in older adults. The Journals of Gerontology: Series A, 72(7), 991-996. https://doi.org/10.1093/gerona/glx065

Haas, E., Hill, R. D., Lambert, M. J., \& Morrell, B. (2002). Do early responders to psychotherapy maintain treatment gains? Journal of Clinical Psychology, 58(9), 11571172. https://doi.org/10.1002/jclp.10044

Hamaker, E. L., Ceulemans, E., Grasman, R. P. P. P., \& Tuerlinckx, F. (2015). Modeling affect dynamics: State of the art and future challenges. Emotion Review, 7(4), 316-322. https://doi.org/10.1177/1754073915590619

Hamaker, E. L., \& Wichers, M. (2017). No time like the present: Discovering the hidden dynamics in intensive longitudinal data. Current Directions in Psychological Science, 26(1), 10-15. https://doi.org/10.1177/0963721416666518

Hamed, K. H., \& Rao, A. R. (1998). A modified Mann-Kendall trend test for autocorrelated data. Journal of Hydrology, 204(1-4), 182-196. https://doi.org/10.1016/S00221694(97)00125-X

Haslbeck, J. M. B., Bringmann, L. F., \& Waldorp, L. J. (2021). A tutorial on estimating timevarying vector autoregressive models. Multivariate Behavioral Research, 56(1), 120149. https://doi.org/10.1080/00273171.2020.1743630

Haslbeck, J. M. B., \& Ryan, O. (2021). Recovering within-person dynamics from psychological time series. Multivariate Behavioral Research. https://doi.org/10.1080/00273171.2021.1896353

Hastings, A., \& Wysham, D. B. (2010). Regime shifts in ecological systems can occur with no warning. Ecology Letters, 13(4), 464-472. https://doi.org/10.1111/j.14610248.2010.01439.x 
Hayes, A. M., \& Andrews, L. A. (2020). A complex systems approach to the study of change in psychotherapy. BMC Medicine, 18(1), 1-13. https://doi.org/10.1186/s12916-020$01662-2$

Hayes, A. M., Laurenceau, J.-P., Feldman, G., Strauss, J. L., \& Cardaciotto, L. (2007). Change is not always linear: The study of nonlinear and discontinuous patterns of change in psychotherapy. Clinical Psychology Review, 27(6), 715-723. https://doi.org/10.1016/j.cpr.2007.01.008

Hayes, A. M., \& Strauss, J. L. (1998). Dynamic systems theory as a paradigm for the study of change in psychotherapy: An application to cognitive therapy for depression. Journal of Consulting and Clinical Psychology, 66(6), 939-947. https://doi.org/10.1037/0022006X.66.6.939

Hayes, A. M., Yasinski, C., Barnes, J. B., \& Bockting, C. L. H. (2015). Network destabilization and transition in depression: New methods for studying the dynamics of therapeutic change. Clinical Psychology Review, 41, 27-39. https://doi.org/10.1016/j.cpr.2015.06.007

Heinzel, S., Tominschek, I., \& Schiepek, G. (2014). Dynamic patterns in psychotherapyDiscontinuous changes and critical instabilities during the treatment of obsessive compulsive disorder. Nonlinear Dynamics, Psychology, and Life Sciences, 18(2), 155176.

Helmich, M. A. (2020). Duration-adjusted Reliable Change Index (DaRCI): Defining clinically relevant symptom changes of varying durations. PsyArXiv. https://doi.org/10.31234/osf.io/q7ch9

Helmich, M. A., Olthof, M., Oldehinkel, A. J., Wichers, M., Bringmann, L. F., \& Smit, A. C. (2021). Early warning signals and critical transitions in psychopathology: Challenges and recommendations. Current Opinion in Psychology, 41, 51-58. https://doi.org/10.1016/j.copsyc.2021.02.008

Helmich, M. A., Snippe, E., Kunkels, Y. K., Riese, H., Smit, A. C., \& Wichers, M. (2020a). Transitions in Depression (TRANS-ID) Recovery: Study protocol for a repeated intensive longitudinal $\mathrm{n}=1$ study design to search for personalized early warning signals of critical transitions towards improvement in depression. Open Science Framework. https://doi.org/10.31234/osf.io/fertq

Helmich, M. A., Wichers, M., Olthof, M., Strunk, G., Aas, B., Aichhorn, W., Schiepek, G., \& Snippe, E. (2020b). Sudden gains in day-to-day change: Revealing nonlinear patterns 
of individual improvement in depression. Journal of Consulting and Clinical Psychology, 88(2), 119-127. https://doi.org/10.1037/ccp0000469

Hosenfeld, B., Bos, E. H., Wardenaar, K. J., Conradi, H. J., van der Maas, H. L. J., Visser, I., \& de Jonge, P. (2015). Major depressive disorder as a nonlinear dynamic system: Bimodality in the frequency distribution of depressive symptoms over time. BMC Psychiatry, 15(1), 222. https://doi.org/10.1186/s12888-015-0596-5

Husen, K., Rafaeli, E., Rubel, J. A., Bar-Kalifa, E., \& Lutz, W. (2016). Daily affect dynamics predict early response in CBT: Feasibility and predictive validity of EMA for outpatient psychotherapy. Journal of Affective Disorders, 206, 305-314. https://doi.org/10.1016/J.JAD.2016.08.025

Ilardi, S. S., \& Craighead, W. E. (1994). The role of nonspecific factors in cognitive-behavior therapy for depression. Clinical Psychology: Science and Practice, 1(2), 138-155. https://doi.org/10.1111/j.1468-2850.1994.tb00016.x

Jacobson, N. S., \& Truax, P. (1991). Clinical significance: A statistical approach to defining meaningful change in psychotherapy research. Journal of Consulting and Clinical Psychology, 59(1), 12-19. https://doi.org/10.1037/0022-006X.59.1.12

Jeronimus, B. F. (2019). Dynamic system perspectives on anxiety and depression. In E. S. Kunnen, N. M. P. de Ruiter, B. F. Jeronimus, \& M. A. van der Gaag (Eds.), Psychosocial Development in Adolescence: Insights from the Dynamic Systems Approach (1st ed.). Routledge Psychology.

Kéfi, S., Dakos, V., Scheffer, M., Van Nes, E. H., \& Rietkerk, M. (2013). Early warning signals also precede non-catastrophic transitions. Oikos, 122(5), 641-648. https://doi.org/10.1111/j.1600-0706.2012.20838.x

Kelley, M. E., Dunlop, B. W., Nemeroff, C. B., Lori, A., Carrillo-Roa, T., Binder, E. B., Kutner, M. H., Rivera, V. A., Craighead, W. E., \& Mayberg, H. S. (2018). Response rate profiles for major depressive disorder: Characterizing early response and longitudinal nonresponse. Depression and Anxiety, 35(10), 992-1000. https://doi.org/10.1002/da.22832

Kunkels, Y. K., Riese, H., Knapen, S. E., Lek, R. F. R. der, George, S. V., Roon, A. M. van, Schoevers, R. A., \& Wichers, M. (2021). Efficacy of early warning signals and spectral periodicity for predicting transitions in bipolar patients: An actigraphy study. Translational Psychiatry, 11(1), 1-10. https://doi.org/10.1038/s41398-021-01465-w 
Kuppens, P., Oravecz, Z., \& Tuerlinckx, F. (2010). Feelings change: Accounting for individual differences in the temporal dynamics of affect. Journal of Personality and Social Psychology, 99(6), 1042-1060. https://doi.org/10.1037/a0020962

Kuranova, A., Booij, S. H., Menne-Lothmann, C., Decoster, J., Van Winkel, R., Delespaul, P., De Hert, M., Derom, C., Thiery, E., Rutten, B. P. F., Jacobs, N., Van Os, J., Wigman, J. T. W., \& Wichers, M. (2020). Measuring resilience prospectively as the speed of affect recovery in daily life: A complex systems perspective on mental health. BMC Medicine, 18(1), 1-11. https://doi.org/10.1186/s12916-020-1500-9

Lever, J. J., Leemput, I. A. van de, Weinans, E., Quax, R., Dakos, V., Nes, E. H. van, Bascompte, J., \& Scheffer, M. (2020). Foreseeing the future of mutualistic communities beyond collapse. Ecology Letters, 23(1), 2-15. https://doi.org/10.1111/ele.13401

Liu, R., Chen, P., Aihara, K., \& Chen, L. (2015). Identifying early-warning signals of critical transitions with strong noise by dynamical network markers. Scientific Reports, 5(1), 17501. https://doi.org/10.1038/srep17501

Lutz, W., Ehrlich, T., Rubel, J., Hallwachs, N., Röttger, M.-A., Jorasz, C., Mocanu, S., Vocks, S., Schulte, D., \& Tschitsaz-Stucki, A. (2013). The ups and downs of psychotherapy: Sudden gains and sudden losses identified with session reports. Psychotherapy Research, 23(1), 14-24. https://doi.org/10.1080/10503307.2012.693837

Molenaar, P. C. M., \& Campbell, C. G. (2009). The new person-specific paradigm in psychology. Current Directions in Psychological Science, 18(2), 112-117. https://doi.org/10.1111/j.1467-8721.2009.01619.x

Nazarimehr, F., Jafari, S., Perc, M., \& Sprott, J. C. (2020). Critical slowing down indicators. EPL, 132(1), 18001. https://doi.org/10.1209/0295-5075/132/18001

Nordberg, S. S., Castonguay, L. G., Fisher, A. J., Boswell, J. F., \& Kraus, D. (2014). Validating the rapid responder construct within a practice research network. Journal of Clinical Psychology, 70(9), 886-903. https://doi.org/10.1002/jclp.22077

Olthof, M., Hasselman, F., Strunk, G., Aas, B., Schiepek, G., \& Lichtwarck-Aschoff, A. (2020a). Destabilization in self-ratings of the psychotherapeutic process is associated with better treatment outcome in patients with mood disorders. Psychotherapy Research, 30(4), 520-531. https://doi.org/10.1080/10503307.2019.1633484

Olthof, M., Hasselman, F., Strunk, G., van Rooij, M., Aas, B., Helmich, M. A., Schiepek, G., \& Lichtwarck-Aschoff, A. (2020b). Critical fluctuations as an early-warning signal for sudden gains and losses in patients receiving psychotherapy for mood disorders. 
Clinical Psychological $\quad$ Science, $\quad 8(1), \quad 25-35$. https://doi.org/10.1177/2167702619865969

Patterson, A. C., Strang, A. G., \& Abbott, K. C. (2021). When and where we can expect to see early warning signals in multispecies systems approaching tipping points: Insights from theory. The American Naturalist, 198(1), E12-E26. https://doi.org/10.1086/714275

Peeters, F., Berkhof, J., Delespaul, P., Rottenberg, J., \& Nicolson, N. A. (2006). Diurnal mood variation in major depressive disorder. Emotion, 6(3), 383-391. https://doi.org/10.1037/1528-3542.6.3.383

Posner, J., Russell, J. A., \& Peterson, B. S. (2005). The circumplex model of affect: An integrative approach to affective neuroscience, cognitive development, and psychopathology. Development and Psychopathology, 17(3), 715-734. https://doi.org/10.1017/S0954579405050340

Riese, H., \& Wichers, M. (2019). Comment on: Eronen MI (2019). The levels problem in psychopathology. Psychological Medicine, 1-2. https://doi.org/10.1017/S0033291719003623

Rubel, J., Lutz, W., Kopta, S. M., Köck, K., Minami, T., Zimmermann, D., \& Saunders, S. M. (2015). Defining early positive response to psychotherapy: An empirical comparison between clinically significant change criteria and growth mixture modeling. Psychological Assessment, 27(2), 478-488. https://doi.org/10.1037/pas0000060

Rush, A. J., Carmody, T., \& Reimitz, P.-E. (2000). The Inventory of Depressive Symptomatology (IDS): Clinician (IDS-C) and Self-Report (IDS-SR) ratings of depressive symptoms. International Journal of Methods in Psychiatric Research, 9(2), 45-59. https://doi.org/10.1002/mpr.79

Scheffer, M. (2009). Critical transitions in nature and society. Princeton University Press. https://press.princeton.edu/titles/8950.html

Scheffer, M., Carpenter, S. R., Lenton, T. M., Bascompte, J., Brock, W., Dakos, V., Van De Koppel, J., Van De Leemput, I. A., Levin, S. A., \& Van Nes, E. H. (2012). Anticipating critical transitions. Science, 338(6105), 344-348. https://doi.org/10.1126/science. 1225244

Schiepek, G. (2009). Complexity and nonlinear dynamics in psychotherapy. European Review, 17(02), 331. https://doi.org/10.1017/S1062798709000763

Schiepek, G., Aichhorn, W., \& Schöller, H. J. (2017). Monitoring change dynamics: A nonlinear approach to psychotherapy feedback. Chaos and Complexity Letters, 11(3), $355-375$. 
Schiepek, G., Heinzel, S., Karch, S., Plöderl, M., \& Strunk, G. (2016). Synergetics in psychology: Patterns and pattern transitions in human change processes. In G. Wunner \& A. Pelster (Eds.), Selforganization in Complex Systems: The Past, Present, and Future of Synergetics (pp. 181-208). Springer International Publishing. http://link.springer.com/10.1007/978-3-319-27635-9_12

Schiepek, G., Schöller, H., de Felice, G., Steffensen, S. V., Bloch, M. S., Fartacek, C., Aichhorn, W., \& Viol, K. (2020). Convergent validation of methods for the identification of psychotherapeutic phase transitions in time series of empirical and model systems. Frontiers in Psychology, 11(1970), 1-14. https://doi.org/10.3389/fpsyg.2020.01970

Schiepek, G., Tominschek, I., \& Heinzel, S. (2014). Self-organization in psychotherapy: Testing the synergetic model of change processes. Frontiers in Psychology, 5. https://doi.org/10.3389/fpsyg.2014.01089

Schreuder, M. J., Hartman, C. A., George, S. V., Menne-Lothmann, C., Decoster, J., van Winkel, R., Delespaul, P., De Hert, M., Derom, C., Thiery, E., Rutten, B. P. F., Jacobs, N., van Os, J., Wigman, J. T. W., \& Wichers, M. (2020). Early warning signals in psychopathology: What do they tell? BMC Medicine, 18(1), 1-12. https://doi.org/10.1186/s12916-020-01742-3

Schreuder, M. J., Hartman, C. A., Groen, R. N., Smit, A. C., Wichers, M., \& Wigman, J. T. W. (in press). Anticipating transitions in mental health in at-risk youth: A six-month daily diary study into early warning signals. Clinical Psychological Science.

Schreuder, M. J., Wigman, J. T. W., Groen, R. N., Weinans, E., Wichers, M., \& Hartman, C. A. (2022). Anticipating the direction of symptom progression using critical slowing down: A proof-of-concept study. BMC Psychiatry, 22(1), 49. https://doi.org/10.1186/s12888022-03686-9

Sedano-Capdevila, A., Porras-Segovia, A., Bello, H. J., Baca-García, E., \& Barrigon, M. L. (2021). Use of ecological momentary assessment to study suicidal thoughts and behavior: A systematic review. Current Psychiatry Reports, 23(7), 41. https://doi.org/10.1007/s11920-021-01255-7

Shalom, J. G., \& Aderka, I. M. (2020). A meta-analysis of sudden gains in psychotherapy: Outcome and moderators. Clinical Psychology Review, 76, 101827. https://doi.org/10.1016/j.cpr.2020.101827

Shapiro, Y. (2015). Dynamical Systems Therapy (DST): Theory and practical applications. Psychoanalytic Dialogues, 25(1), 83-107. https://doi.org/10.1080/10481885.2015.991245 
Smit, A. C., Helmich, M. A., Bringmann, L. F., Oldehinkel, A. J., Wichers, M., \& Snippe, E. (n.d.). Critical slowing down in momentary affect as early warning signal of impending transitions in depression. Submitted.

Smits, I. A. M., Timmerman, M. E., Barelds, D. P. H., \& Meijer, R. R. (2015). The Dutch Symptom Checklist-90-Revised: Is the use of the subscales justified? European Journal of Psychological Assessment, 31(4), 263-271. https://doi.org/10.1027/1015$5759 / \mathrm{a} 000233$

Strunk, G., \& Lichtwarck-Aschoff, A. (2019). Therapeutic chaos. Journal for Person-Oriented Research, 5(2), 81-100. https://doi.org/10.17505/jpor.2019.08

Stulz, N., Lutz, W., Leach, C., Lucock, M., \& Barkham, M. (2007). Shapes of early change in psychotherapy under routine outpatient conditions. Journal of Consulting and Clinical Psychology, 75(6), 864-674. https://doi.org/10.1037/0022-006X.75.6.864

Tang, T. Z., \& DeRubeis, R. J. (1999). Sudden gains and critical sessions in cognitivebehavioral therapy for depression. Journal of Consulting and Clinical Psychology, 67(6), 894-904. https://doi.org/10.1037/0022-006X.67.6.894

Thompson, R. J., Mata, J., Jaeggi, S. M., Buschkuehl, M., Jonides, J., \& Gotlib, I. H. (2012). The everyday emotional experience of adults with major depressive disorder: Examining emotional instability, inertia, and reactivity. Journal of Abnormal Psychology, 121(4), 819-829. https://doi.org/10.1037/a0027978

van de Leemput, I. A., Wichers, M., Cramer, A. O. J., Borsboom, D., Tuerlinckx, F., Kuppens, P., van Nes, E. H., Viechtbauer, W., Giltay, E. J., Aggen, S. H., Derom, C., Jacobs, N., Kendler, K. S., van der Maas, H. L. J., Neale, M. C., Peeters, F., Thiery, E., Zachar, P., \& Scheffer, M. (2014). Critical slowing down as early warning for the onset and termination of depression. Proceedings of the National Academy of Sciences of the United States of America, 111(1), 87-92. https://doi.org/10.1073/pnas.1312114110

Van Der Bolt, B., van Nes, E. H., \& Scheffer, M. (2021). No warning for slow transitions. Journal of the Royal Society, Interface, 18(176), 20200935. https://doi.org/10.1098/rsif.2020.0935

van Nes, E. H., Arani, B. M. S., Staal, A., van der Bolt, B., Flores, B. M., Bathiany, S., \& Scheffer, M. (2016). What do you mean, 'tipping point'? Trends in Ecology \& Evolution, 31(12), 902-904. https://doi.org/10.1016/j.tree.2016.09.011

Vittengl, J. R., Clark, L. A., Thase, M. E., \& Jarrett, R. B. (2016). Defined symptom-change trajectories during acute-phase cognitive therapy for depression predict better 
longitudinal outcomes. Behaviour Research and Therapy, 87, 48-57. https://doi.org/10.1016/j.brat.2016.08.008

Wichers, M. (2014). The dynamic nature of depression: A new micro-level perspective of mental disorder that meets current challenges. Psychological Medicine, 44(7), 13491360. https://doi.org/10.1017/S0033291713001979

Wichers, M., Groot, P. C., Psychosystems, ESM Group, \& EWS Group. (2016). Critical slowing down as a personalized early warning signal for depression. Psychotherapy and Psychosomatics, 85(2), 114-116. https://doi.org/10.1159/000441458

Wichers, M., Schreuder, M. J., Goekoop, R., \& Groen, R. N. (2019). Can we predict the direction of sudden shifts in symptoms? Transdiagnostic implications from a complex systems perspective on psychopathology. Psychological Medicine, 49(3), 380-387. https://doi.org/10.1017/S0033291718002064

Wichers, M., Smit, A. C., \& Snippe, E. (2020). Early warning signals based on momentary affect dynamics can expose nearby transitions in depression: A confirmatory singlesubject time-series study. Journal for Person-Oriented Research, 6(1), 1-15. https://doi.org/10.17505/jpor.2020.22042

Wichers, M., Wigman, J. T. W., \& Myin-Germeys, I. (2015). Micro-level affect dynamics in psychopathology viewed from complex dynamical system theory. Emotion Review, 7(4), 362-367. https://doi.org/10.1177/1754073915590623

Wood, S. N. (2017). Generalized additive models: An introduction with R. In Generalized Additive Models: An Introduction with R, Second Edition (2nd ed.). CRC Press. https://doi.org/10.1201/9781315370279

Wright, A. G. C., \& Woods, W. C. (2020). Personalized models of psychopathology. Annual Review of Clinical Psychology, 16, 49-74. https://doi.org/10.1146/annurev-clinpsy102419

Yik, M. S. M., Russell, J. A., \& Feldman Barrett, L. (1999). Structure of self-reported current affect: Integration and beyond. Journal of Personality and Social Psychology, 77(3), 600-619. https://doi.org/10.1037/0022-3514.77.3.600

Zeeman, E. C. (1976). Catastrophe theory. Scientific American, 234(4), 65-83. 


\title{
Supplementary Material to
}

\section{'Detecting impending symptom transitions using early warning signals in individuals receiving treatment for depression'}

\author{
Marieke A. Helmich, Arnout C. Smit, Laura F. Bringmann, Marieke J. Schreuder,
} Albertine J. Oldehinkel, Marieke Wichers, Evelien Snippe

\section{Brief introduction}

In this Supplementary Material, we show various additional plots and tables to provide further insight into the effects of different model settings (sensitivity checks) on our results, using different confidence levels for the transition identification, and the findings of our further pre-registered exploratory work.

With the low number of transition cases, it is prudent to interpret the proportion of EWS in the groups and for individual variables with caution, and we recommend looking mainly at overall patterns.

For the comparison of the analyses at different confidence levels, it is also worth noting that there is relevant overlap between the groups at different thresholds, which may affect the number of false positive and false negative EWS. For those who change transition status, the analysis is conducted as if they have no transition - over their entire time series.

\section{Moving Window method}

\subsection{Main analysis}

The main moving window analysis was conducted with transitions identified at 95\% confidence level (CL), a minimally relevant effect size of $\tau \geq .1$, a window size of 70 observations, outliers winsorized and within-window detrending. 


\section{Figure S1a}

Density plot of the $t$-values found in the moving window analysis of the autocorrelation

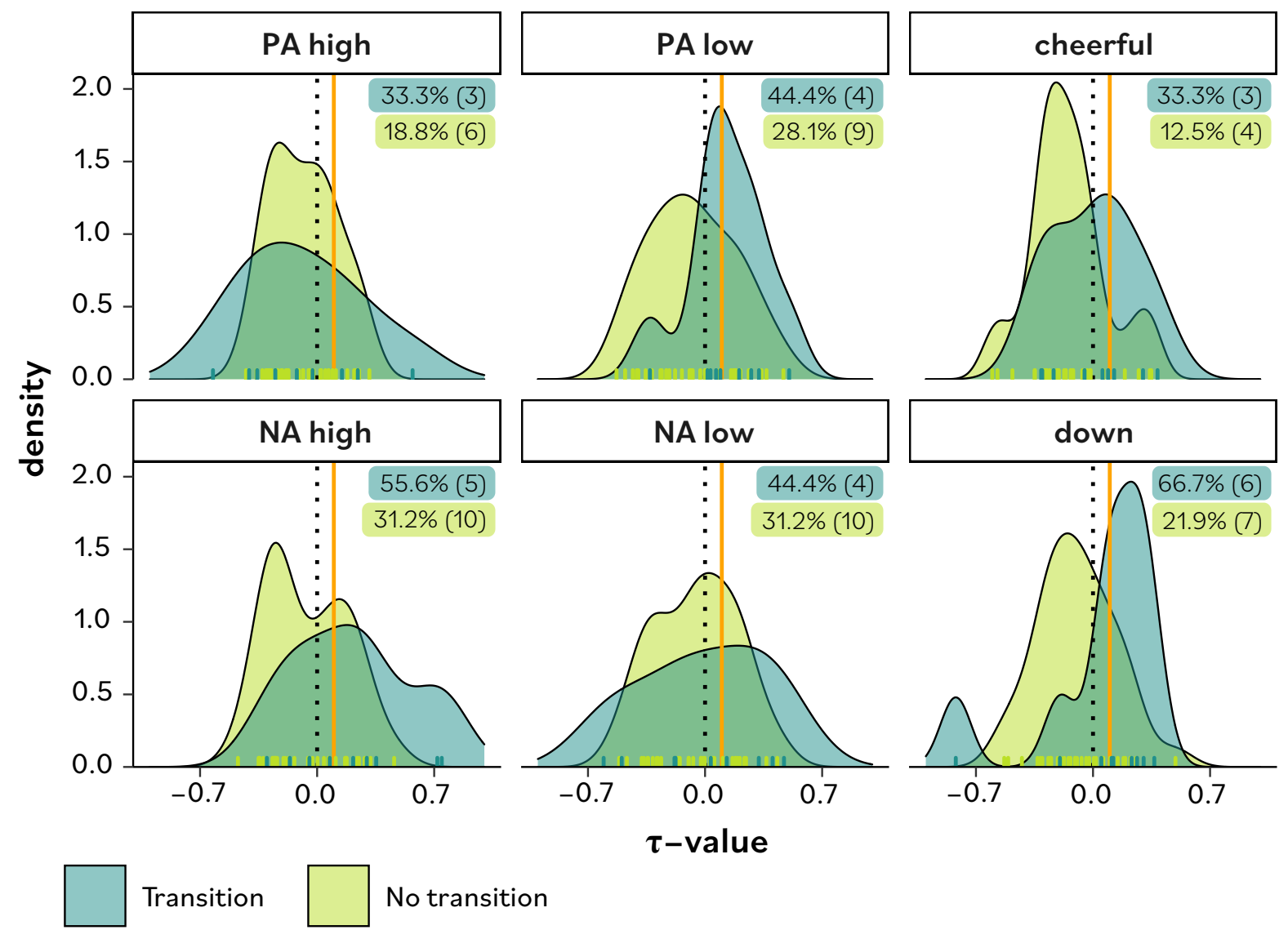

Note. PA = Positive Affect; NA = Negative Affect; high = high arousal; low = low arousal. Note that group sizes were unequal: $N=9$ individuals with a transition, and $N=32$ without a transition, at a $95 \%$ confidence threshold. For this analysis, individual time series were winsorized, detrended within windows of 70 observations. Number of early warning signals indicated in the upper right corner of each plot for both groups, meet the criterion of $\tau \geq .1$ The dotted vertical line indicates 0 , and the orange vertical line indicates the $\tau \geq .1$ minimally relevant effect size threshold.

\section{Distribution of $\mathrm{\tau}$ for the autocorrelation}

In Figure S1a, we can see that the distributions of the $\tau$ showed different spreads and peaks in the two groups - with the modes in the transition group appearing relatively more on the right (higher, more positive values of the $\mathrm{t}$ ), and the modes of the notransition group appearing generally closer to zero or below. Furthermore, it is noteworthy that many people showed negative values of $\tau$, indicating significant decreases in autocorrelation over time (both for individuals with and without transitions). 


\section{Figure S1b}

Density plot of the $t$-values found in the moving window analysis of the variance.

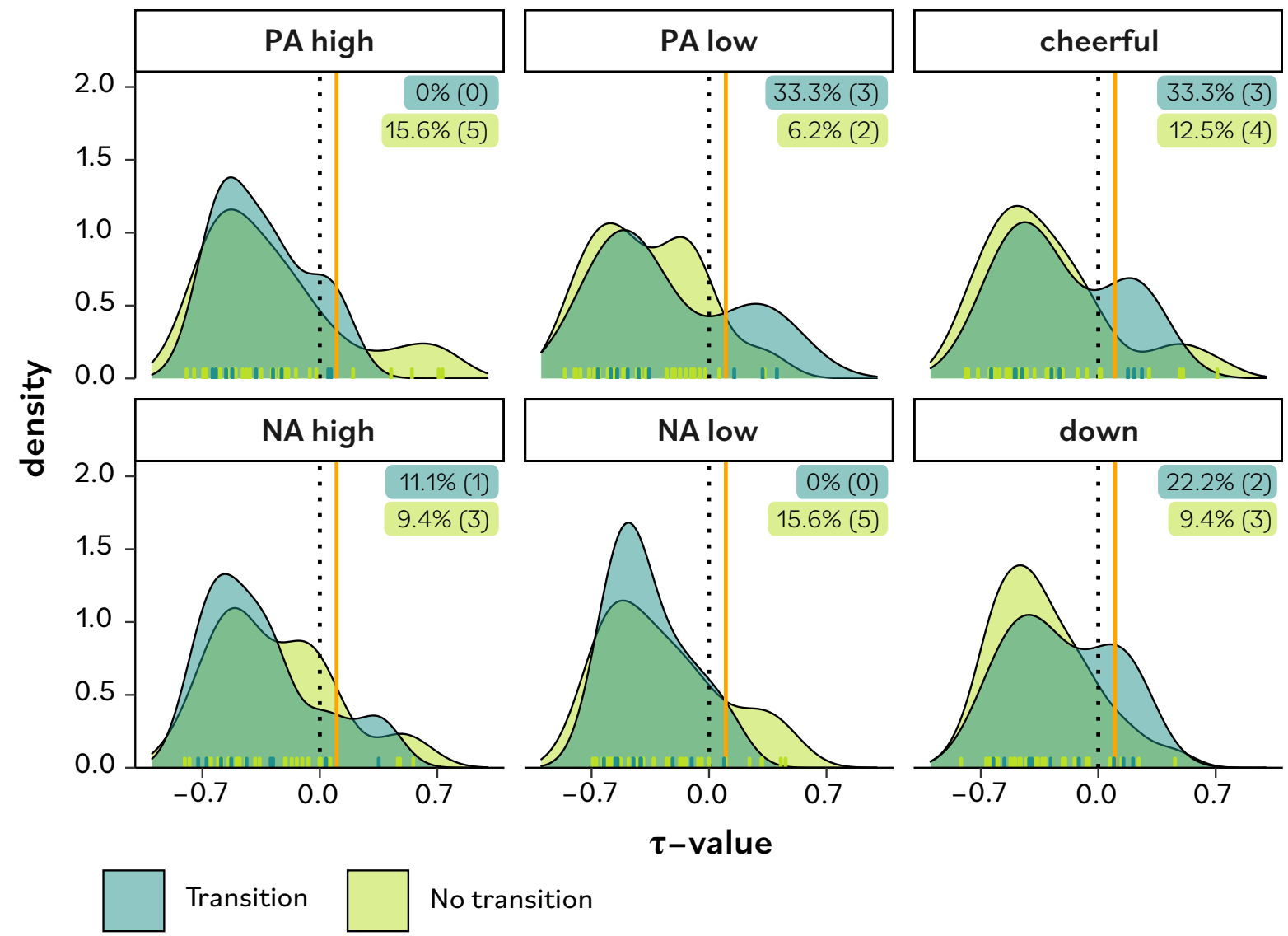

Note. PA = Positive Affect; NA = Negative Affect; high = high arousal; low = low arousal. Note that group sizes were unequal: $N=9$ individuals with a transition, and $N=32$ without a transition, at a $95 \%$ confidence threshold. For this analysis, individual time series were winsorized, detrended within windows of 70 observations. Number of early warning signals indicated in the upper right corner of each plot for both groups, meet the criterion of $\tau \geq .1$ The dotted vertical line indicates 0 , and the orange vertical line indicates the $\tau \geq .1$ minimally relevant effect size threshold.

\section{Distribution of $\boldsymbol{\tau}$ for the variance}

In Figure S1b, we can see that the differences between groups on the six variables is negligible, with the distributions largely overlapping. However, it is noteworthy that in both groups many people showed negative values of $\tau$ : significant decreases in variance over time (prior to a transition, or during the entire study period). For the people with transitions in particular, this is contrary to what we expected from the theory of early warning signals. 


\subsection{Sensitivity checks}

Sensitivity checks were run to check the results of the reference moving window analysis (i.e., window size $n=70$, outliers winsorized and within-window detrend, minimally relevant effect size of $\tau \geq .1$, transition identified at $95 \%$ confidence) against:

- Using significance of the Mann-Kendall test only (no minimally relevant effect size)

- Hamed-Rao window-dependency correction for the Mann-Kendall $p$-value.

- Global detrend

- No winsorizing (outliers unchanged)

- Alternate window sizes: 35 obs, 105 obs, 140 obs (1, 3, and 4 weeks, respectively)

- Excluding cases with transitions to higher symptom levels

- Different transition confidence levels: $90 \%$ and $99 \%$

\section{Altered model settings for the autocorrelation}

Table S1a reveals that the number of individuals with early warning signals in the autocorrelation tended to be higher in the group without transitions, but proportionally (in terms of percentage) the group with transitions consistently showed more EWS across the different sensitivity checks.

Of all the checks, the Hamed-Rao correction for the M-K $p$-value yields the most conservative results: true positives reduced to $25 \%$ across the four circumplex variables (i.e., PA high/low - NA high/low), and the false positives to $12.5 \%$. Conversely, increasing the window size to 140 observations seemed to improve the rate of true positives (from $44.4 \%$ to $58.3 \%$ in the circumplex variables), along with a small increase in false positives (from $27.3 \%$ to $31.2 \%$ in the circumplex variables). However, most model setting changes led to a slight increase or decrease (ca. 2-5\%) in both the true and false positive rates.

Shortening the time series in the non-transition group also appeared to result in an increase in false positive EWS for some variables, particularly for cheerful and the PA variables. 


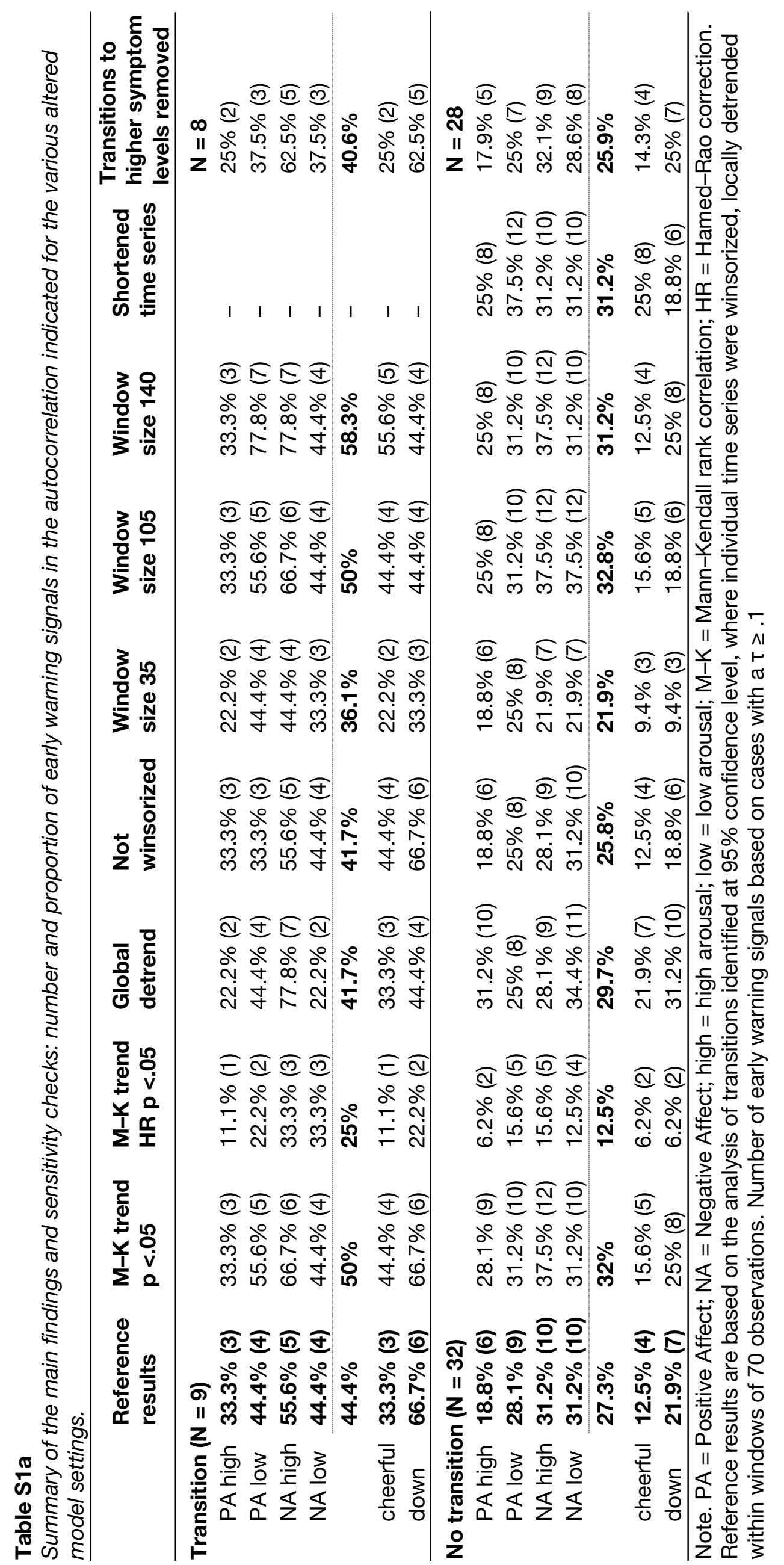


For individual variables, this proportional difference - more true positives than false positives - was almost always maintained in the various analyses (some exceptions occurred in the global detrend analysis in particular), though the percentual differences were not always large.

\section{Altered model settings for the variance}

Across the sensitivity checks shown in Table S1b, the variance showed EWS in only

a few variables for the individuals with transitions (range $=0-33.3 \%$ ), while those without transitions showed at least one EWS in all variables (range $=3.1-21.9 \%$ ). The lack of a proportional difference in EWS between individuals with and without a transition was consistent across the sensitivity checks. Averaged across the affect circumplex variables, true positives ranged from $8.3-16.7 \%$ in the various analyses, and false positives ranged from 10.9-16.4\%. None of the altered model settings led to a relevant change in the results, all showing only a slight increase or decrease (ca. $2-5 \%)$ in both the true and false positive rates.

Like for the autocorrelation, the Hamed-Rao adjusted M-K p-value resulted in the most conservative outcomes, especially for the single items. Conversely, the shortened time series in the non-transition group led to the strongest increase in false positives in the circumplex variables, however, this was still only a $\sim 5 \%$ increase compared to the main analysis. Notably, NA low arousal never showed EWS in the transition group in any of the analyses, while PA low arousal showed EWS in 2 or 3 individuals in the transition group (22.2-33.3\%), with a similar 2 to 4 false positives in the no-transition group (6.2-12.5\%). 


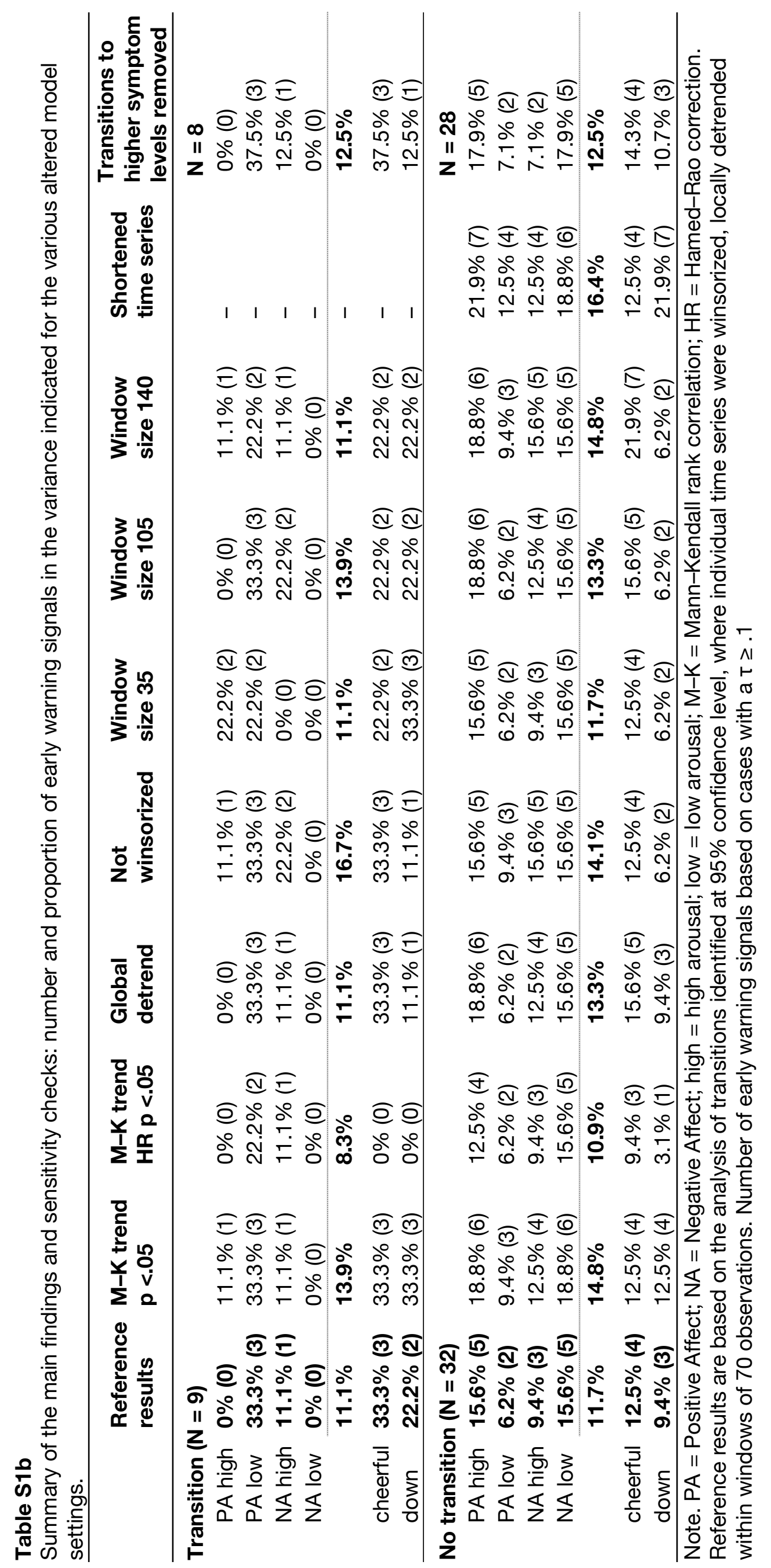




\section{Presence and effect of transitions to higher symptom levels}

Even if we expect therapy to mainly foster symptom improvements, EWS are theoretical indicators of any impending critical transition, including to a worsened state. Therefore, we explored the frequency of transitions towards higher levels of symptoms in our sample post hoc by applying the $\mathrm{DaRCl}$ at $95 \% \mathrm{CL}$ and the stability criterion (for symptom deterioration: the mean of two weeks prior to the shift was at least 8.6 points lower than the mean of the two weeks after the identified transition).

Four cases from the non-transition group experienced shifts toward higher symptom levels. One participant in the transition group had a transition towards worsened symptoms that occurred earlier than a transition towards improvement. As a sensitivity check, we removed these individuals from the main analysis and recalculated the proportion of EWS per affect measure over the remaining individuals. The excluded cases showed a slightly higher percentage of EWS in the autocorrelation, which led to a slight decrease in the percentage of false positives in the recalculation (see Table S1a), and they showed very few EWS in the variance, leading to a slight increase in the false positive percentages (see Table S1b). Overall, removing these cases did not lead to strongly different results.

To explore these transitions to higher symptom levels fully, we recalculated the moving-window EWS for these individuals before the new transitions to higher symptom levels. One participant had fewer than 105 observations before the transition and was therefore excluded from the analysis. For the three remaining individuals that were previously identified as having no transition, one fewer EWS (in PA high) was identified for the autocorrelation, and no difference in the number of EWS was found for the variance compared to the original results. For the participant who showed both transitions towards worsening and improvement of symptoms, the recalculation of EWS before the earlier deterioration led to four fewer EWS in the autocorrelation (PA high and low, and the two single items; the EWS in NA low remained), and three new EWS in the variance (in PA high, NA low and single item 'cheerful'), while one EWS was lost (in single item 'down').

In short, only few transitions towards higher symptom levels were found in our sample. After recalculation of the moving window analysis those cases showed a similar number of EWS, though in different variables compared to their results in the 
main analysis. Note that due to the small sample size, findings should be cautiously interpreted and no conclusions can be drawn regarding how these results generalize to other patients experiencing transitions towards higher levels of depressive symptoms (for a dedicated study of that research question, see footnote ${ }^{1}$ ).

\section{Moving window results at different transition thresholds}

Table S2 details the proportion of EWS found in the moving window analyses for the autocorrelation and variance when transitions were defined at a lower confidence level (90\%), and at a higher confidence level (99\%), compared to the main analysis (95\%). Across the analyses for the autocorrelation, the group with transitions

\section{Table S2}

Comparison of the number and proportion of early warning signals in the autocorrelation and variance found in the moving window analysis when different confidence levels were used to identify transitions.

\begin{tabular}{|c|c|c|c|c|c|c|c|}
\hline & \multirow[b]{2}{*}{ Variable } & \multicolumn{3}{|c|}{ Autocorrelation } & \multicolumn{3}{|c|}{ Variance } \\
\hline & & $90 \% \mathrm{CL}$ & $95 \% \mathrm{CL}$ & $99 \% \mathrm{CL}$ & $90 \% \mathrm{CL}$ & $95 \% \mathrm{CL}$ & $99 \% \mathrm{CL}$ \\
\hline \multirow[t]{7}{*}{ Transition } & PA high & $50 \%(7)$ & $33.3 \%(3)$ & $50 \%(3)$ & $7.1 \%(1)$ & $0 \%(0)$ & $16.7 \%(1)$ \\
\hline & PA low & $50 \%(7)$ & $44.4 \%(4)$ & $33.3 \%(2)$ & $21.4 \%(3)$ & $33.3 \%(3)$ & $16.7 \%(1)$ \\
\hline & NA high & $35.7 \%(5)$ & $55.6 \%(5)$ & $66.7 \%(4)$ & $14.3 \%(2)$ & $11.1 \%(1)$ & $16.7 \%(1)$ \\
\hline & NA low & $35.7 \%(5)$ & $44.4 \%(4)$ & $50 \%(3)$ & $7.1 \%(1)$ & $0 \%(0)$ & $16.7 \%(1)$ \\
\hline & & $46.2 \%$ & $44.4 \%$ & $50 \%$ & $13.5 \%$ & $11.1 \%$ & $16.7 \%$ \\
\hline & cheerful & $42.9 \%(6)$ & $33.3 \%(3)$ & $33.3 \%(2)$ & $28.6 \%(4)$ & $33.3 \%(3)$ & $16.7 \%(1)$ \\
\hline & down & $57.1 \%(8)$ & $66.7 \%(6)$ & $50 \%(3)$ & $35.7 \%(5)$ & $22.2 \%(2)$ & $16.7 \%(1)$ \\
\hline \multirow[t]{7}{*}{ No transition } & PA high & $18.5 \%(5)$ & $18.8 \%(6)$ & $17.1 \%(6)$ & $18.5 \%(5)$ & $15.6 \%(5)$ & $14.3 \%(5)$ \\
\hline & PA low & $33.3 \%(9)$ & $28.1 \%(9)$ & $31.4 \%(11)$ & $7.4 \%(2)$ & $6.2 \%(2)$ & $8.6 \%(3)$ \\
\hline & NA high & $33.3 \%(9)$ & $31.2 \%(10)$ & $31.4 \%(11)$ & $11.1 \%(3)$ & $9.4 \%(3)$ & $14.3 \%(5)$ \\
\hline & NA low & $33.3 \%(9)$ & $31.2 \%(10)$ & $28.6 \%(10)$ & $14.8 \%(4)$ & $15.6 \%(5)$ & $17.1 \%(6)$ \\
\hline & & $22.2 \%$ & $27.3 \%$ & $27.1 \%$ & $13 \%$ & $11.7 \%$ & $13.6 \%$ \\
\hline & cheerful & $11.1 \%(3)$ & $12.5 \%(4)$ & $14.3 \%(5)$ & $14.8 \%(4)$ & $12.5 \%(4)$ & $17.1 \%(6)$ \\
\hline & down & $18.5 \%(5)$ & $21.9 \%(7)$ & $20 \%(7)$ & $11.1 \%(3)$ & $9.4 \%$ (3) & $8.6 \%(3)$ \\
\hline
\end{tabular}

Note. EWS = Early warning signals; $\mathrm{CL}=$ confidence level; $\mathrm{PA}=$ Positive Affect, NA = Negative Affect; high $=$ high arousal; low $=$ low arousal. Total $N=41$. At $90 \% \mathrm{CL}: 13$ transitions (one person had insufficient data before the transition to be analysed). At 95\% CL: 9 transitions. At 99\% CL: 6 transitions. Main analysis at $95 \% \mathrm{CL}$ in bold. For the other CLs, all other analysis settings were equal: individual time series were winsorized, locally detrended within windows of 70 observations. Number of early warning signals based on cases with a $\tau \geq .1$

\footnotetext{
${ }^{1}$ Smit, A. C., Helmich, M. A., Bringmann, L. F., Oldehinkel, A. J., Wichers, M., \& Snippe, E. (Submitted). Detecting impending symptom transitions using early warning signals in individuals who discontinue antidepressant medication.
} 
(consisting of 14 individuals at $90 \% \mathrm{CL}$, and 6 individuals at $99 \% \mathrm{CL}$ ), the proportion of identified EWS was consistently higher than in the group of individuals without a transition - with the exception of NA high and NA low arousal at $90 \%$ CL, and PA low arousal at $99 \% \mathrm{CL}$, where the proportion of EWS was similar for both groups. For the variance, the lack of proportional difference in EWS between groups also extended to these alternate analyses.

\subsection{Further exploration}

\subsubsection{Combining EWS}

The moving window results were further explored to see if the number of EWS per individual could be a simple way to improve the specificity of the results. We would expect that individuals with transitions show EWS in multiple variables in both autocorrelation and variance (4 variables $\times 2$ EWS), whereas individuals without transitions would show no or fewer EWS.

\section{Differentiating groups by number of EWS per person}

Based on Figure S2, we see that the individuals with a transition all $(N=9)$ showed at least one EWS - out of a possible 8 - in one of the four variables (PA high/low - NA high/low), in either the autocorrelation or variance results of the moving window analysis. In the group without transitions, most people $(N=19)$ had 0 or only 1 EWS, but there were also 13 individuals with at least two EWS.

Thus, combining EWS and setting the threshold to a minimum of two EWS per person, results in $77.8 \%$ true positives, and $40.6 \%$ false positives. Raising the threshold by one step (number of EWS $\geq 3$ ) lowers the sensitivity again, capturing $33.3 \%$ true positives, and $18.8 \%$ false positives. 


\section{Figure S2}

Total number of EWS per person in autocorrelation and variance combined, indicated by the moving window analysis.

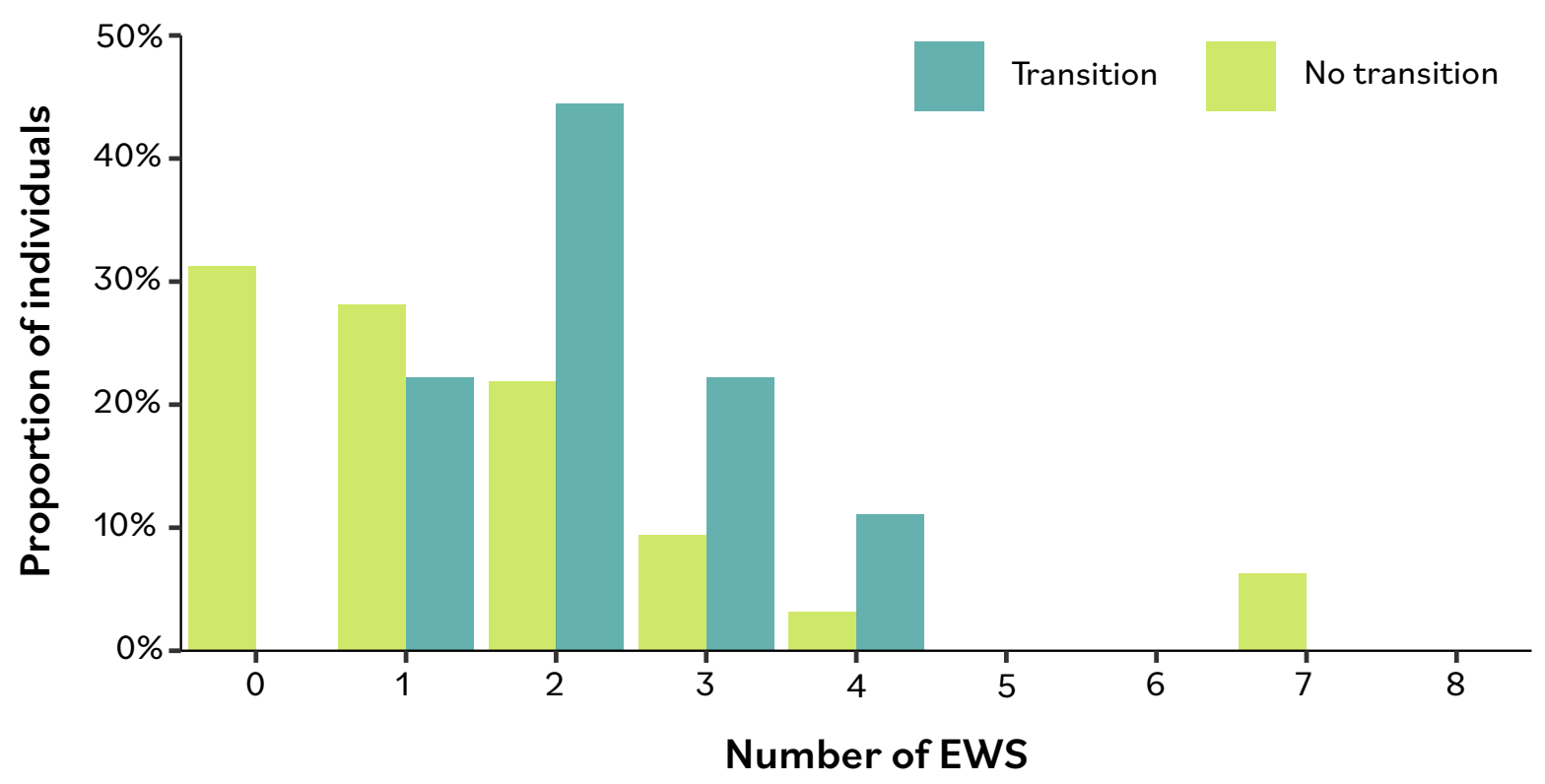

Note. $N=9$ individuals with a transition, and $N=32$ without a transition.

Figure S3

Number of EWS per person in autocorrelation and variance separately, indicated by the moving window analysis.

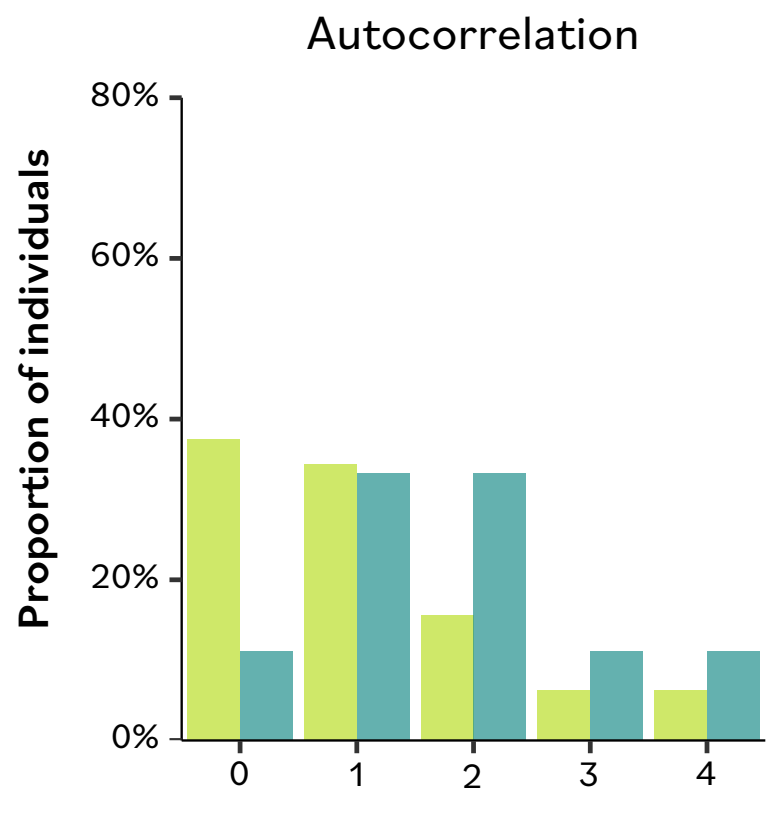

Number of EWS

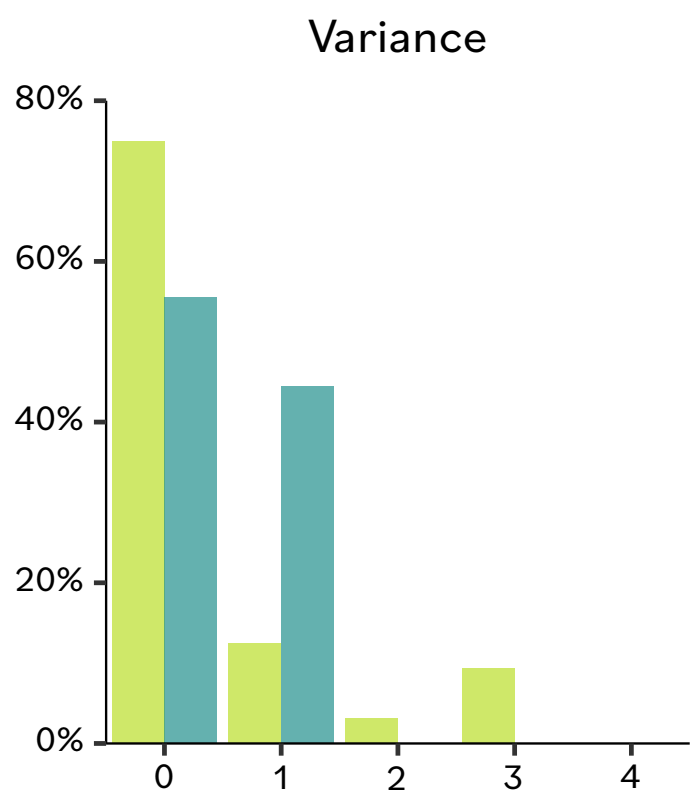

Number of EWS

Transition No transition

Note. $N=9$ individuals with a transition, and $N=32$ without a transition. 


\section{Number of EWS per person in autocorrelation and variance separately}

On the left side of Figure S3, we see that all but one (thus, 88.89\%) of the individuals with a transition showed at least one EWS for the autocorrelation (out of a possible 4), compared to $62.5 \%$ in the group without transitions. Raising the minimum number of EWS in autocorrelation by one (number of EWS $\geq 2$ ) leads to $55.5 \%$ true positives from the transition group, and $28.1 \%$ false positives. For minimally 3 EWS in autocorrelation, the transition group shows $22.2 \%$ true positives, while the notransition group shows $12.5 \%$ false positives.

For the variance, on the right side of Figure S3, 4 out of 9 (44.4\%) individuals with a transition had one variable (out of a possible 4) with EWS, compared to $8(25 \%)$ in the group without transitions. The remaining people with transitions had no EWS in the variance at all (i.e., $55.5 \%$ false negatives), while $12.5 \%$ of non-transitioning individuals was still found with a threshold number of EWS $\geq 2$.

\subsubsection{MRES: $\tau$ exploration}

As indicated in the preregistration, we examined different minimally relevant effect sizes (MRES) than the chosen $\tau$ of .1, to compare the balance of true positives (EWS in individuals with a transition) to false positive EWS (EWS in individuals with no transition) at different increments of $\tau$ in the moving window results. To that end, we calculated the balanced accuracy (BA; the average of the proportions of correct classifications) for the autocorrelation and variance, as follows:

1. Sensitivity or True positive rate (TPR): Number of EWS in the transition group/ $N$ transition group

2. Specificity or True negative rate (TNR): 1 - (Number of EWS in the nontransition group/ $\mathrm{N}$ non-transition group)

3. Balanced accuracy (BA): TPR + TNR / 2

\section{Balanced accuracy for different increments of $\tau$}

Figure S4 shows the balanced accuracy for the EWS found in autocorrelation and variance with the moving window method. For the $B A$, a value of $50 \%$ means the rate of true positives to true negatives is equal, no better than chance; values below $50 \%$ 
indicate that there are more false positives or false negatives; values above $50 \%$ indicate a higher rates of true positives or negatives. We can see that across the various increments of $\tau$, some variables remain stable in the trade-off between sensitivity and specificity indicated by the BA percentages (e.g., PA high and NA low in autocorrelation, and NA high and NA low in variance), whereas others have a higher $B A$ at some thresholds of $\tau$. For instance, the single item "down" seems to find a higher BA at a $\tau$ between 0 and 0.2 , for both the autocorrelation and variance. Also note that for the autocorrelation, NA high appears to work relatively well across all increments. However, overall, this figure shows the BA values for our sample are modest at best, and particularly the variance performs close to chance. Given these low BA values and the inconsistent 'optimal' threshold for different variables, it is not possible to make any strong recommendations about appropriate $\tau$ thresholds for future research.

\section{Figure S4}

Balanced Accuracy for the autocorrelation and variance results of the moving window analysis
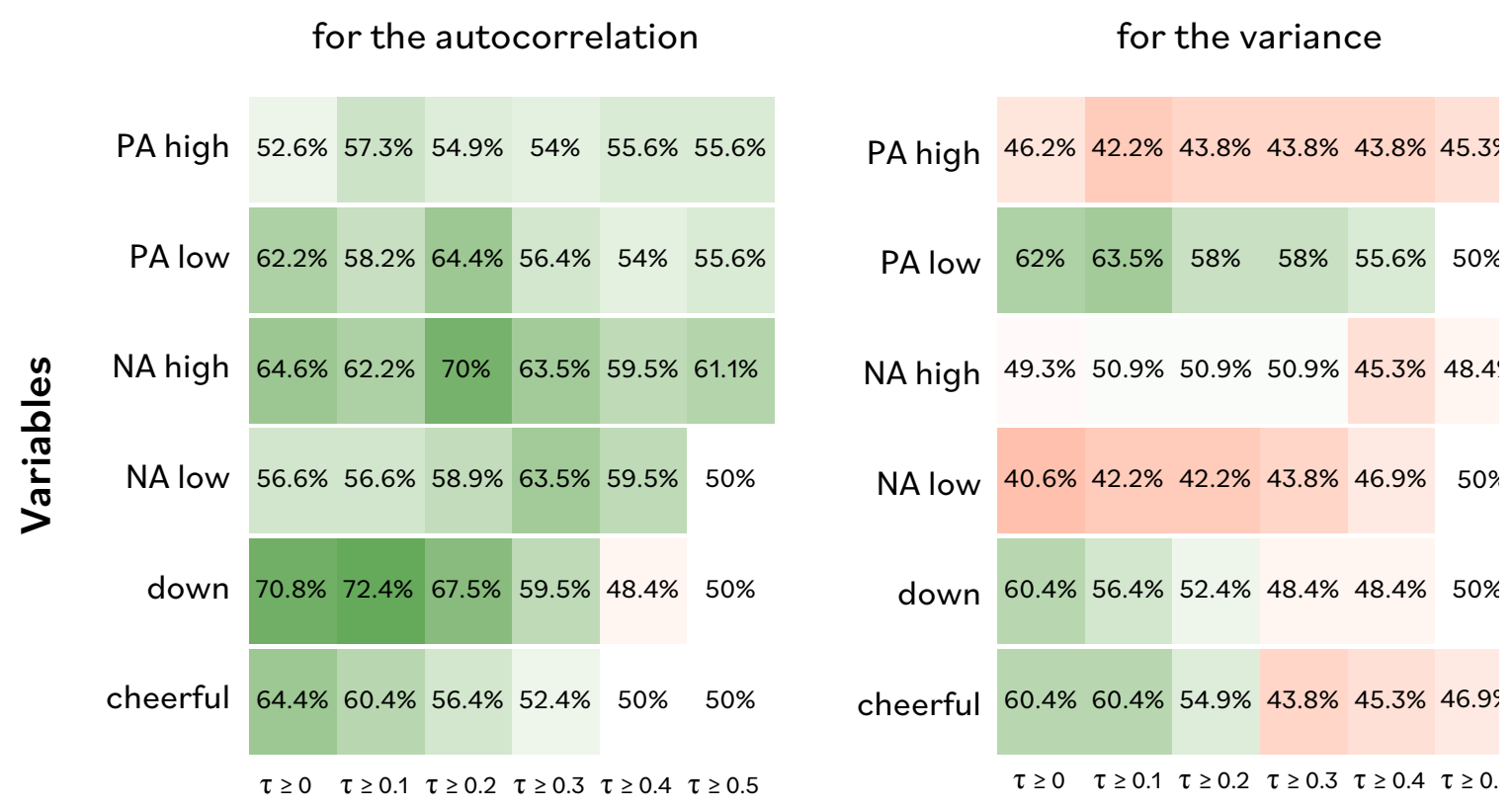

\section{minimally relevant effect size}

Note. $\mathrm{PA}=$ Positive Affect; NA = Negative Affect; high = high arousal; low = low arousal. $N=9$ individuals with a transition, and $N=32$ without a transition. Balanced accuracy (BA): True Positive Rate + True Negative Rate / 2 


\section{TV-AR GAM method}

\subsection{Main analysis and sensitivity check}

The main TV-AR GAM analysis results show the different time-varying patterns that were found for the transition group, and the group without a transition (columns in bold in the table below). Additional sensitivity checks were conducted to examine the effect of the longer time series in the no-transition group, and the analysis was repeated with the transitions identified at $90 \%$ and $99 \%$ confidence level.

\section{TV-AR-GAM results for different $\mathrm{CL}$ and shortened time series}

Table S3 details the proportion of EWS found in the autocorrelation, as modeled by the TV-AR-GAM method, when transitions were defined at a lower confidence level $(90 \%)$, and at a higher confidence level (99\%), compared to the main analysis (95\%).

Across these analyses at different confidence levels, the number of cases with a significant increase in autocorrelation is similar in the transition group and nontransition group and remarkably low. At 90\%, 95\% and 99\%, the transition group had $6 \%, 5.6 \%$ and $0 \%$ EWS across the four affect circumplex variables, respectively, while the individuals without transitions showed $3.1 \%, 3.1 \%$ and $3.3 \%$ significant increases in autocorrelation across those four variables.

Another pattern across analyses, variables and the two groups, was that half the individuals or more (range $=48 \%$ to $87 \%$ ) did not show a significant change over time at all.

Among the significant smooth curves, the proportion of cases with nonlinear changes (i.e., cubic, quadratic, or more complex curves) stands out. For instance, summarized across the four affect circumplex variables, $27.8 \%$ of the significant changes were nonlinear in the $95 \% \mathrm{CL}$ transition group, and $25.8 \%$ in the nontransition group. The percentages at the other confidence levels were $10.7 \%$ and $25.3 \%$ at $90 \% \mathrm{CL}$, and $22.2 \%$ and $29 \%$ at $99 \% \mathrm{CL}$ for the transition and no-transition groups, respectively.

Of further note are the results of the sensitivity check of the effect of time series length for individuals without transitions. For all confidence levels, the shortened time series led to fewer significant smooth curves, particularly to fewer cases with a decrease. Likely, the loss in significant smooth curves is because the TV-AR GAM 
had less data available in the shortened time series and could not fit the $95 \%$ credible intervals as narrowly as it could for the full-length time series. Wider credible intervals would result in fewer cases where change could be identified with certainty.

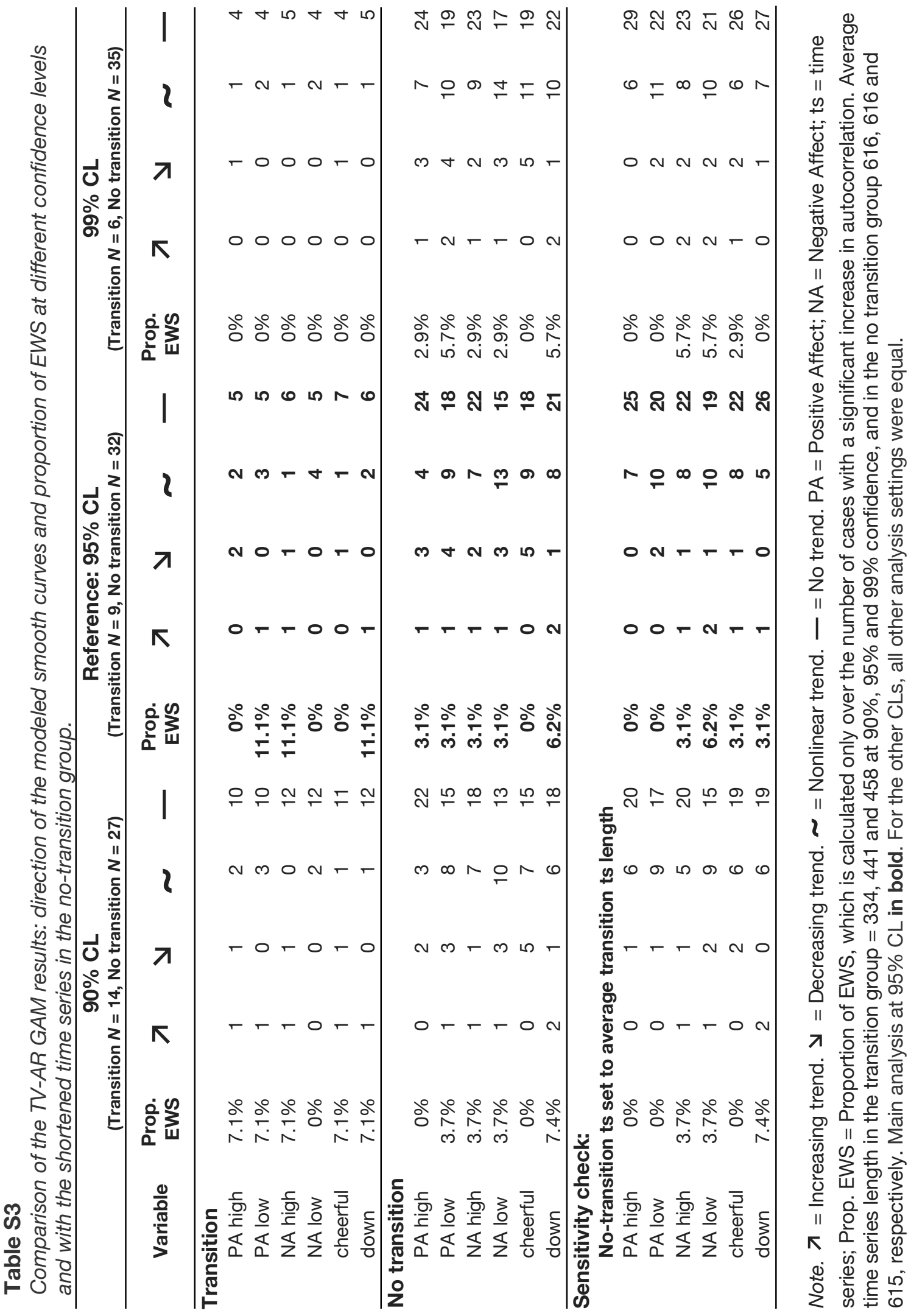




\section{Confirmatory factor analysis}

We conducted a post hoc multilevel confirmatory factor analysis to examine whether the four theory-based ${ }^{2}$ composite affective circumplex items (NA/PA $x$ high/low arousal) were reliable constructs. Only the first two weeks (70 observations maximum per person) of data were entered into the model so that we could assume that the measured constructs were relatively stable during that period. We used the cfa function from the lavaan $\mathrm{R}$ package version $0.6-10^{3}$ with default settings to test the item loadings for each of the four factors at Level 1 (within person), and Level 2 (between persons). We also estimated cross-correlations between all four factors.

The overall model fit appeared to be adequate ${ }^{4}$ : the comparative fit index (CFI; optimally $>0.95$ ) of 0.943 and root mean square error of approximation (RMSEA; optimally $<0.06)$ of $0.058,90 \% \mathrm{Cl}[0.054,0.062]$ indicate an acceptable fit between the chosen model and the observed data. The standardized root mean square residual $(S R M R)$ indicated good fit $(<0.08)$ for both the within-person covariance matrix $(S R M R=0.047)$ and the between-person covariance matrix $(S R M R=0.078)$.

The item and factor level results of this analysis are reported in Table S4. Individual item loadings at the within-person level ranged from 0.47 (moderate) to 0.87 (very high), and from 0.75 (high) to 1.00 (highest) at the between-person level. Thus, the items corresponded well to the chosen factor structure. Between the factors, the correlations also appeared quite large, with the within-person correlations ranging between -0.38 (low moderate) and 0.84 (high), and the between-person factor correlations indicating -0.47 (moderate) to 0.92 (very high). The relatively high factor intercorrelations, particularly for PA high, PA low and NA low, suggest that perhaps a simpler factor structure would be possible - e.g., with a single PA factor however, we did not formally test this.

\footnotetext{
${ }^{2}$ Posner, J., Russell, J. A., \& Peterson, B. S. (2005). The circumplex model of affect: An integrative approach to affective neuroscience, cognitive development, and psychopathology. Development and Psychopathology, 17(3), 715-734. https://doi.org/10.1017/S0954579405050340

${ }^{3}$ Yves Rosseel (2012). lavaan: An R Package for Structural Equation Modeling. Journal of Statistical Software, 48(2), 1-36. https://doi.org/10.18637/jss.v048.i02

${ }^{4} \mathrm{Hu}$, L. T., \& Bentler, P. M. (1999). Cutoff criteria for fit indexes in covariance structure analysis: Conventional criteria versus new alternatives. Structural equation modeling: a multidisciplinary journal, 6(1), 1-55. https://doi.org/10.1080/10705519909540118
} 


\section{Table S4}

Results of a multilevel Confirmatory Factor Analysis of the four composite affect circumplex factors

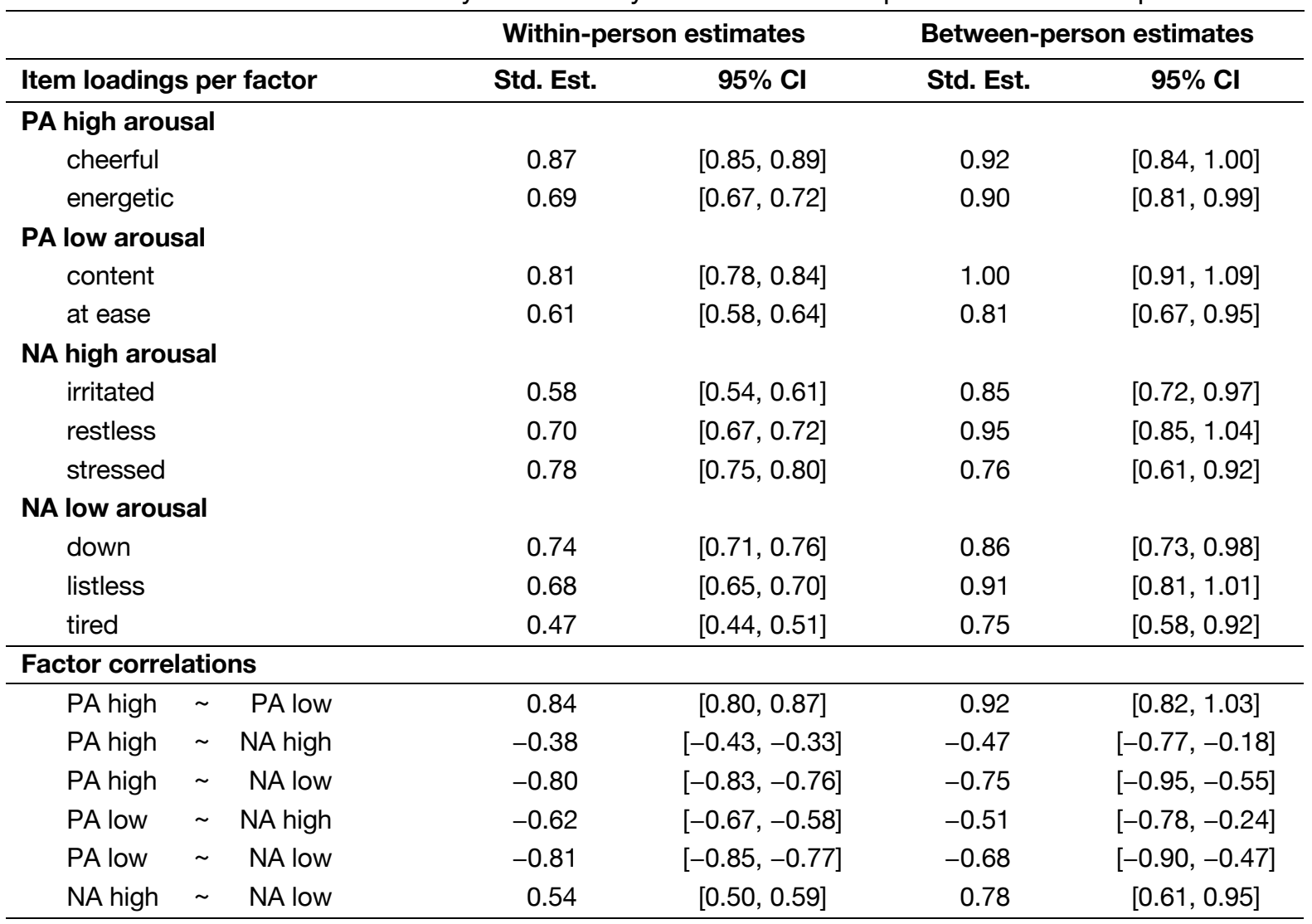

Note. Level $1=2505$ observations, Level $2=41$ participants. Std. Est. $=$ Standardized estimates. $\mathrm{Cl}=$ Confidence Interval. $\mathrm{PA}=$ Positive affect. $\mathrm{NA}=$ Negative affect. 